INTERNATIONAL MONETARY FUND

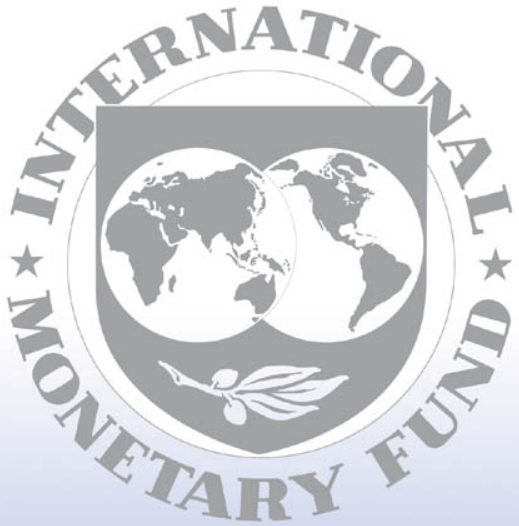

Staff






\section{Bangladesh: Statistical Appendix}

This Statistical Appendix for Bangladesh was prepared by a staff team of the International Monetary Fund as background documentation for the periodic consultation with the member country. It is based on the information available at the time it was completed on April 18, 2007. The views expressed in this document are those of the staff team and do not necessarily reflect the views of the government of Bangladesh or the Executive Board of the IMF.

The policy of publication of staff reports and other documents by the IMF allows for the deletion of market-sensitive information.

To assist the IMF in evaluating the publication policy, reader comments are invited and may be sent by e-mail to publicationpolicy@imf.org.

Copies of this report are available to the public from

International Monetary Fund • Publication Services

700 19th Street, N.W. • Washington, D.C. 20431

Telephone: (202) 6237430 • Telefax: (202) 6237201

E-mail: publications@imf.org • Internet: http://www.imf.org

Price: $\$ 18.00$ a copy

\section{International Monetary Fund Washington, D.C.}


This page intentionally left blank

(CInternational Monetary Fund. Not for Redistribution 
INTERNATIONAL MONETARY FUND

\section{BANGLADESH}

\section{Statistical Appendix}

Prepared by Wafa Abdelati and Erik Lueth (APD)

Approved by the Asia and Pacific Department

June 1, 2007

Contents

Page

1. Gross Domestic Product at Current Prices, 1999/00-2005/06

2. Gross Domestic Product at Constant Prices, 1999/00-2005/06 ………..........................

3. Gross Domestic Product by Expenditure at Current Prices, 1999/00-2005/06................. $\underline{5}$

4. Manufacturing Production by Main Categories, 1999/00-2005/06 …………................. $\underline{6}$

5. Energy Statistics, 1999/00-2005/06 ………......................................................

6. Retail Prices of Petroleum Products, 1999/00-2006/07 ……….....................................

7. Consumer Price Index, National, 1999/00-2004/05 ..................................................

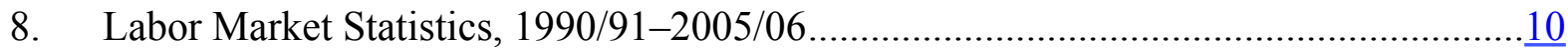

9. Central Government Operations, 2000/01-2005/06 ....................................................

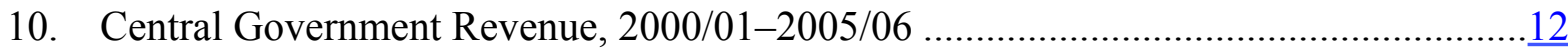

11. Central Government Current Expenditure, 2000/01-2005/06 ....................................13

12. Central Government Food Account, 1999/00-2005/06 ...............................................

13. Nonfinancial Public Corporations Operations, 2000/01-2005/06 …….........................15

14. Profits of Nonfinancial Public Corporations, 2000/01-2005/06 ……...........................16



16. Central Bank Balance Sheet, 2001/02-2006/07 …....................................................

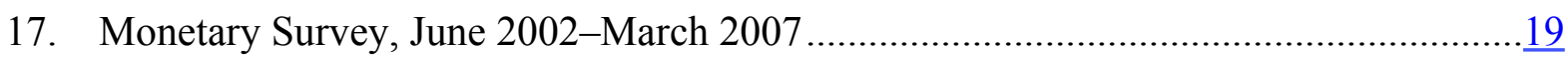

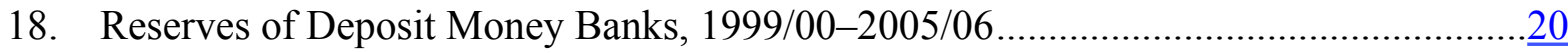

19. Interest Rate Structure of the Banking Sector, 2002/03-2006/07 ..................................

20. Interest Rate Structure of Nonbank Financial Institutions and Government Debt,

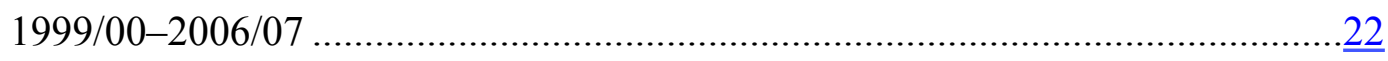

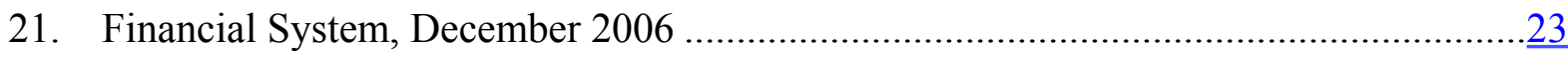

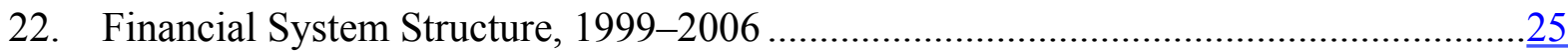

23. Summary Performance Indicators in the Banking System, 1999-2006 …......................26

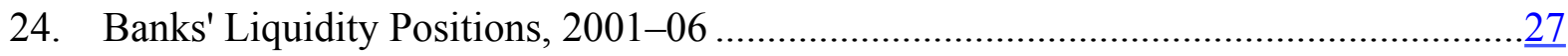

25. Banks' Foreign Exchange Positions, 1999-2006 ……............................................... 
26. Bank's Reported Capital, 2001-06

27. Balance of Payments, 2000/01-2005/06.

28. Exports by Main Product Categories, 2000/01-2005/06 .............................................

29. Imports by Main Product Categories, 2000/01-2005/06 .............................................

30. Geographical Distribution of Foreign Trade, 1999/00-2005/06 …................................33

31. Worker's Remittances by Country of Origin, 2001/02-2006/07 …….........................34

32. Services Account, 2000/01-2005/06 …………..................................................

33. External Aid Commitments and Disbursements, 1999/00-2005/06...............................

34. External Aid Disbursements by Donors, 1999/00-2005/06 ……………………….....

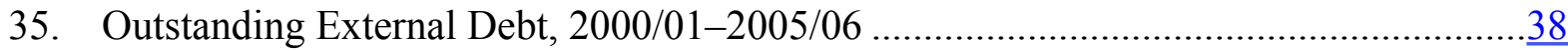

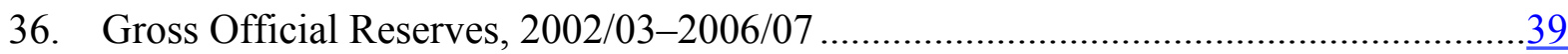

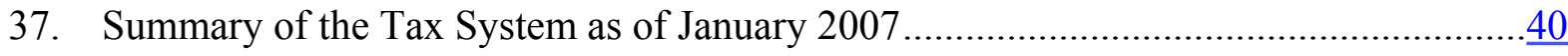


Table 1. Bangladesh: Gross Domestic Product at Current Prices, 1999/00-2005/06 1/

(In billions of taka)

\begin{tabular}{|c|c|c|c|c|c|c|c|}
\hline & 1999/00 & $2000 / 01$ & $2001 / 02$ & $2002 / 03$ & $2003 / 04$ & $2004 / 05$ & $2005 / 062 /$ \\
\hline Agriculture and forestry & 447 & 456 & 460 & 488 & 524 & 562 & 622 \\
\hline Crops and horticulture & 334 & 341 & 339 & 360 & 389 & 415 & 461 \\
\hline Animal farming & 69 & 69 & 71 & 75 & 79 & 87 & 97 \\
\hline Forest and related services & 44 & 47 & 50 & 53 & 56 & 60 & 64 \\
\hline Fishing & 137 & 134 & 139 & 143 & 148 & 155 & 163 \\
\hline Mining and quarrying & 23 & 26 & 30 & 33 & 36 & 40 & 46 \\
\hline Natural gas and crude petroleum & 13 & 15 & 17 & 19 & 21 & 23 & 26 \\
\hline Other mining and quarrying & 10 & 11 & 13 & 14 & 16 & 17 & 21 \\
\hline Manufacturing & 348 & 382 & 418 & 458 & 515 & 588 & 689 \\
\hline Large- and medium-scale & 249 & 273 & 296 & 324 & 364 & 415 & 490 \\
\hline Small-scale & 99 & 109 & 122 & 134 & 152 & 173 & 199 \\
\hline Electricity, gas, and water & 31 & 33 & 36 & 40 & 44 & 49 & 54 \\
\hline Electricity & 26 & 28 & 31 & 33 & 37 & 41 & 45 \\
\hline Gas & 3 & 4 & 4 & 4 & 5 & 5 & 6 \\
\hline Water & 1 & 2 & 2 & 2 & 3 & 3 & 3 \\
\hline Construction & 176 & 193 & 212 & 230 & 254 & 291 & 328 \\
\hline Wholesale and retail trade & 292 & 325 & 353 & 391 & 441 & 503 & 570 \\
\hline Hotels and restaurants & 15 & 16 & 17 & 19 & 22 & 25 & 29 \\
\hline Transport, storage, and communication & 197 & 221 & 255 & 311 & 344 & 383 & 432 \\
\hline Land transport & 145 & 161 & 189 & 241 & 269 & 294 & 328 \\
\hline Water transport & 25 & 26 & 27 & 28 & 29 & 30 & 31 \\
\hline Air transport & 4 & 4 & 4 & 4 & 4 & 5 & 5 \\
\hline Support transport services, storage & 8 & 9 & 10 & 10 & 11 & 12 & 13 \\
\hline Post and telecommunications & 16 & 21 & 25 & 28 & 32 & 43 & 55 \\
\hline Financial intermediation & 36 & 39 & 42 & 47 & 52 & 59 & 67 \\
\hline Bank & 28 & 30 & 32 & 35 & 39 & 45 & 50 \\
\hline Insurance & 7 & 8 & 9 & 10 & 11 & 13 & 14 \\
\hline Others & 2 & 2 & 2 & 2 & 2 & 2 & 3 \\
\hline Real estate, renting, and business activities & 211 & 224 & 240 & 257 & 276 & 297 & 322 \\
\hline Public administration and defense & 62 & 67 & 71 & 78 & 86 & 96 & 110 \\
\hline Education & 54 & 59 & 64 & 71 & 79 & 88 & 99 \\
\hline Health and social works & 54 & 57 & 61 & 66 & 72 & 81 & 90 \\
\hline Community, social, and personal services & 204 & 217 & 237 & 267 & 300 & 339 & 383 \\
\hline GDP at current market prices & 2,371 & 2,535 & 2,732 & 3,005 & 3,330 & 3,707 & 4,157 \\
\hline
\end{tabular}

Source: Bangladesh Bureau of Statistics.

1/ Fiscal year ending June 30.

2/ Preliminary estimate. 
Table 2. Bangladesh: Gross Domestic Product at Constant Prices, 1999/00-2005/06 1/

(Annual percent change)

\begin{tabular}{|c|c|c|c|c|c|c|c|}
\hline & $1999 / 00$ & $2000 / 01$ & $2001 / 02$ & $2002 / 03$ & $2003 / 04$ & $2004 / 05$ & $2005 / 062 /$ \\
\hline Agriculture and forestry & 6.9 & 5.5 & -0.6 & 3.3 & 4.4 & 1.8 & 5.2 \\
\hline Crops and horticulture & 8.1 & 6.2 & -2.4 & 2.9 & 4.3 & 0.2 & 5.0 \\
\hline Animal farming & 2.7 & 2.8 & 4.7 & 4.5 & 5.0 & 7.2 & 6.2 \\
\hline Forest and related services & 4.9 & 4.9 & 4.9 & 4.4 & 4.2 & 5.1 & 5.2 \\
\hline Fishing & 8.9 & -4.5 & 2.2 & 2.3 & 3.1 & 3.7 & 3.9 \\
\hline Mining and quarrying & 9.5 & 9.8 & 4.5 & 7.2 & 7.7 & 8.4 & 9.3 \\
\hline Natural gas and crude petroleum & 14.6 & 14.0 & 4.9 & 8.9 & 9.0 & 9.0 & 9.5 \\
\hline Other mining and quarrying & 3.5 & 4.2 & 4.0 & 4.7 & 5.7 & 7.4 & 8.8 \\
\hline Manufacturing & 4.8 & 6.7 & 5.5 & 6.8 & 7.1 & 8.2 & 10.8 \\
\hline Large and medium scale & 4.9 & 6.6 & 4.6 & 6.6 & 7.0 & 8.3 & 11.4 \\
\hline Small scale & 5.8 & 7.0 & 7.7 & 7.2 & 7.5 & 7.9 & 9.2 \\
\hline Electricity, gas, and water & 6.8 & 7.4 & 7.6 & 8.0 & 9.1 & 8.9 & 7.7 \\
\hline Electricity & 6.9 & 7.6 & 7.8 & 7.3 & 9.2 & 8.6 & 7.5 \\
\hline Gas & 5.6 & 6.1 & 6.5 & 8.8 & 8.8 & 8.9 & 9.4 \\
\hline Water & 8.1 & 7.1 & 7.5 & 20.0 & 8.0 & 14.4 & 7.5 \\
\hline Construction & 8.5 & 8.7 & 8.6 & 8.1 & 8.3 & 8.3 & 8.3 \\
\hline Wholesale and retail trade & 7.3 & 6.4 & 6.6 & 6.1 & 6.6 & 7.1 & 6.8 \\
\hline Hotels and restaurants & 6.9 & 7.0 & 6.9 & 7.0 & 7.1 & 7.1 & 7.5 \\
\hline Transport, storage, and communication & 6.1 & 7.9 & 6.6 & 6.9 & 6.2 & 7.9 & 8.0 \\
\hline Land transport & 6.3 & 6.4 & 6.7 & 6.6 & 6.0 & 4.3 & 4.1 \\
\hline Water transport & 1.8 & 0.6 & 0.3 & 0.1 & 0.2 & 2.0 & 2.0 \\
\hline Air transport & 16.8 & 4.6 & -16.8 & -1.4 & 0.8 & 2.5 & 5.3 \\
\hline Support transport services, storage & 13.2 & 18.1 & -4.6 & -1.2 & -1.4 & 2.9 & 6.1 \\
\hline Post and telecommunications & 5.6 & 26.9 & 20.9 & 17.9 & 14.6 & 31.8 & 26.7 \\
\hline Financial intermediation & 5.5 & 5.5 & 6.7 & 6.7 & 7.0 & 8.9 & 8.5 \\
\hline Bank & 3.9 & 4.0 & 5.5 & 5.9 & 6.7 & 9.1 & 8.2 \\
\hline Insurance & 13.1 & 13.5 & 12.4 & 10.3 & 8.1 & 8.3 & 9.2 \\
\hline Others & 5.5 & 0.0 & 2.1 & 2.3 & 6.9 & 8.5 & 10.9 \\
\hline Real estate, renting, and business activities & 3.8 & 3.4 & 3.4 & 3.5 & 3.6 & 3.7 & 3.7 \\
\hline Public administration and defense & 6.0 & 5.9 & 5.9 & 5.2 & 7.1 & 8.0 & 8.2 \\
\hline Education & 7.7 & 7.1 & 7.6 & 7.6 & 7.7 & 7.9 & 9.1 \\
\hline Health and social works & 4.8 & 4.9 & 5.3 & 5.6 & 6.2 & 7.4 & 7.8 \\
\hline Community, social, and personal services & 3.1 & 3.2 & 3.2 & 3.3 & 4.0 & 4.1 & 4.1 \\
\hline GDP at constant market prices & 5.9 & 5.3 & 4.4 & 5.3 & 6.3 & 6.0 & 6.6 \\
\hline
\end{tabular}

Source: Bangladesh Bureau of Statistics.

1/ Data based on 1995/96 benchmark and prices; fiscal year ending June 30.

2/ Preliminary estimate. 
Table 3. Bangladesh: Gross Domestic Product by Expenditure at Current Prices, 1999/00-2005/06 1/

\begin{tabular}{|c|c|c|c|c|c|c|c|}
\hline & $1999 / 00$ & $2000 / 01$ & $2001 / 02$ & $2002 / 03$ & $2003 / 04$ & $2004 / 05$ & $2005 / 062 /$ \\
\hline & \multicolumn{7}{|c|}{ (In billions of taka) } \\
\hline $\begin{array}{l}\text { Consumption } \\
\text { Private } \\
\text { General government }\end{array}$ & $\begin{array}{r}1,947 \\
1,839 \\
108\end{array}$ & $\begin{array}{r}2,079 \\
1,965 \\
114\end{array}$ & $\begin{array}{r}2,236 \\
2,099 \\
137\end{array}$ & $\begin{array}{r}2,446 \\
2,285 \\
161\end{array}$ & $\begin{array}{r}2,679 \\
2,495 \\
184\end{array}$ & $\begin{array}{r}2,965 \\
2,760 \\
205\end{array}$ & $\begin{array}{r}3,316 \\
3,085 \\
230\end{array}$ \\
\hline $\begin{array}{l}\text { Investment } \\
\text { Private } \\
\text { Public }\end{array}$ & $\begin{array}{l}546 \\
370 \\
176\end{array}$ & $\begin{array}{l}586 \\
402 \\
184\end{array}$ & $\begin{array}{l}632 \\
458 \\
174\end{array}$ & $\begin{array}{l}704 \\
517 \\
186\end{array}$ & $\begin{array}{l}800 \\
594 \\
206\end{array}$ & $\begin{array}{l}909 \\
679 \\
230\end{array}$ & $\begin{array}{r}1,025 \\
775 \\
249\end{array}$ \\
\hline Domestic demand & 2,493 & 2,665 & 2,868 & 3,150 & 3,479 & 3,874 & 4,340 \\
\hline $\begin{array}{l}\text { Net exports } \\
\text { Exports of goods and nonfactor services } \\
\text { Imports of goods and nonfactor services }\end{array}$ & $\begin{array}{r}-124 \\
331 \\
456\end{array}$ & $\begin{array}{r}-155 \\
390 \\
545\end{array}$ & $\begin{array}{r}-130 \\
390 \\
520\end{array}$ & $\begin{array}{r}-175 \\
427 \\
602\end{array}$ & $\begin{array}{r}-178 \\
515 \\
693\end{array}$ & $\begin{array}{r}-240 \\
615 \\
854\end{array}$ & $\begin{array}{r}-261 \\
789 \\
1,049\end{array}$ \\
\hline Gross domestic expenditure (GDE) & 2,368 & 2,510 & 2,738 & 2,974 & 3,301 & 3,635 & 4,080 \\
\hline Statistical discrepancy & 2 & 25 & -6 & 32 & 29 & 72 & 78 \\
\hline GDP at current market prices & 2,371 & 2,535 & 2,732 & 3,005 & 3,330 & 3,707 & 4,157 \\
\hline Net factor income from abroad & 87 & 88 & 125 & 166 & 176 & 189 & 272 \\
\hline Gross national income & 2,458 & 2,623 & 2,857 & 3,172 & 3,505 & 3,896 & 4,429 \\
\hline Net current transfers from abroad & 37 & 23 & 19 & 22 & 21 & 27 & 37 \\
\hline Gross disposable national income & 2,495 & 2,646 & 2,876 & 3,193 & 3,526 & 3,923 & 4,466 \\
\hline Gross national saving & 548 & 568 & 640 & 747 & 847 & 958 & 1,150 \\
\hline Gross domestic saving $3 /$ & 421 & 431 & 502 & 529 & 622 & 670 & 764 \\
\hline \multirow[t]{2}{*}{ Gross domestic saving 4/ } & 424 & 456 & 496 & 560 & 650 & 742 & 842 \\
\hline & \multicolumn{7}{|c|}{ (In percent of GDP) } \\
\hline $\begin{array}{l}\text { Consumption } \\
\text { Private } \\
\text { General government }\end{array}$ & $\begin{array}{r}82.1 \\
77.5 \\
4.6\end{array}$ & $\begin{array}{r}82.0 \\
77.5 \\
4.5\end{array}$ & $\begin{array}{r}81.8 \\
76.8 \\
5.0\end{array}$ & $\begin{array}{r}81.4 \\
76.0 \\
5.3\end{array}$ & $\begin{array}{r}80.5 \\
74.9 \\
5.5\end{array}$ & $\begin{array}{r}80.0 \\
74.4 \\
5.5\end{array}$ & $\begin{array}{r}79.8 \\
74.2 \\
5.5\end{array}$ \\
\hline $\begin{array}{l}\text { Investment } \\
\text { Private } \\
\text { Public }\end{array}$ & $\begin{array}{r}23.0 \\
15.6 \\
7.4\end{array}$ & $\begin{array}{r}23.1 \\
15.9 \\
7.3\end{array}$ & $\begin{array}{r}23.1 \\
16.8 \\
6.4\end{array}$ & $\begin{array}{r}23.4 \\
17.2 \\
6.2\end{array}$ & $\begin{array}{r}24.0 \\
17.8 \\
6.2\end{array}$ & $\begin{array}{r}24.5 \\
18.3 \\
6.2\end{array}$ & $\begin{array}{r}24.7 \\
18.7 \\
6.0\end{array}$ \\
\hline Domestic demand & 105.1 & 105.1 & 105.0 & 104.8 & 104.5 & 104.5 & 104.4 \\
\hline $\begin{array}{l}\text { Net exports } \\
\text { Exports of goods and nonfactor services } \\
\text { Imports of goods and nonfactor services }\end{array}$ & $\begin{array}{l}-5.2 \\
14.0 \\
19.2\end{array}$ & $\begin{array}{l}-6.1 \\
15.4 \\
21.5\end{array}$ & $\begin{array}{l}-4.8 \\
14.3 \\
19.0\end{array}$ & $\begin{array}{l}-5.8 \\
14.2 \\
20.0\end{array}$ & $\begin{array}{l}-5.3 \\
15.5 \\
20.8\end{array}$ & $\begin{array}{l}-6.5 \\
16.6 \\
23.0\end{array}$ & $\begin{array}{l}-6.3 \\
19.0 \\
25.2\end{array}$ \\
\hline Statistical discrepancy & 0.1 & 1.0 & -0.2 & 1.1 & 0.9 & 2.0 & 1.9 \\
\hline GDP at current market prices & 100.0 & 100.0 & 100.0 & 100.0 & 100.0 & 100.0 & 100.0 \\
\hline $\begin{array}{l}\text { Memorandum items: } \\
\text { Gross national saving } \\
\text { Gross domestic saving 3/ } \\
\text { Gross domestic saving 4/ }\end{array}$ & $\begin{array}{l}23.1 \\
17.8 \\
17.9\end{array}$ & $\begin{array}{l}22.4 \\
17.0 \\
18.0\end{array}$ & $\begin{array}{l}23.4 \\
18.4 \\
18.2\end{array}$ & $\begin{array}{l}24.9 \\
17.6 \\
18.6\end{array}$ & $\begin{array}{l}25.4 \\
18.7 \\
19.5\end{array}$ & $\begin{array}{l}25.8 \\
18.1 \\
20.0\end{array}$ & $\begin{array}{l}27.7 \\
18.4 \\
20.2\end{array}$ \\
\hline
\end{tabular}

Sources: Bangladesh Bureau of Statistics; and IMF staff estimates.

1/ Fiscal year ending June 30 .

2/ Preliminary estimate.

3/ GDE minus consumption.

4/ GDP minus consumption. 
Table 4. Bangladesh: Manufacturing Production by Main Categories, 1999/00-2005/06 1/

Weights $\quad 1999 / 00 \quad 2000 / 01 \quad 2001 / 02 \quad 2002 / 03 \quad 2003 / 04 \quad 2004 / 05 \quad 2005 / 06$

\begin{tabular}{|c|c|c|c|c|c|c|c|c|}
\hline \multirow[b]{2}{*}{ Total manufacturing } & \multicolumn{8}{|c|}{$($ Volume Index; $1988 / 89=100)$} \\
\hline & 100 & 214 & 228 & 239 & 254 & 266 & 291 & 327 \\
\hline Food, beverages, and tobacco & 22 & 181 & 186 & 215 & 223 & 229 & 250 & 269 \\
\hline $\begin{array}{l}\text { Jute, textiles, and leather } \\
\text { Of which: }\end{array}$ & 38 & 262 & 279 & 278 & 290 & 311 & 340 & 405 \\
\hline Garments & 9 & 766 & 812 & 769 & 805 & 891 & 944 & 1122 \\
\hline Wood products & 0 & 176 & 178 & 182 & 182 & 183 & 202 & 242 \\
\hline Paper and paper products & 5 & 288 & 289 & 307 & 321 & 329 & 371 & 399 \\
\hline Chemicals, fertilizer, petroleum, and rubber & 24 & 185 & 202 & 213 & 246 & 250 & 269 & 284 \\
\hline Nonmetallic products & 3 & 248 & 296 & 312 & 331 & 344 & 389 & 418 \\
\hline Basic metal products & 2 & 170 & 188 & 197 & 205 & 209 & 236 & 252 \\
\hline \multirow[t]{2}{*}{ Fabricated metal products } & 6 & 98 & 103 & 109 & 109 & 112 & 132 & 144 \\
\hline & \multicolumn{8}{|c|}{ (Annual percentage change) } \\
\hline Total manufacturing & & 4.9 & 6.6 & 4.5 & 6.6 & 4.5 & 9.4 & 12.5 \\
\hline Food, beverages, and tobacco & & 7.1 & 3.2 & 15.4 & 3.6 & 2.7 & 8.9 & 7.8 \\
\hline $\begin{array}{l}\text { Jute, textiles, and leather } \\
\text { Of which: }\end{array}$ & & 5.8 & 6.7 & -0.6 & 4.3 & 7.3 & 9.4 & 19.2 \\
\hline Garments & & 9.5 & 5.9 & -5.3 & 4.7 & 10.7 & 5.9 & 18.9 \\
\hline Wood products & & 4.6 & 0.9 & 2.5 & 0.2 & 0.4 & 10.1 & 19.8 \\
\hline Paper and paper products & & 1.1 & 0.2 & 6.3 & 4.7 & 2.5 & 12.6 & 7.5 \\
\hline Chemicals, fertilizer, petroleum, and rubber & & 1.9 & 9.3 & 5.6 & 15.4 & 1.6 & 7.3 & 5.8 \\
\hline Nonmetalic products & & 22.4 & 19.4 & 5.4 & 6.2 & 4.0 & 13.0 & 7.4 \\
\hline Basic metal products & & 4.2 & 10.7 & 4.7 & 3.8 & 2.0 & 12.9 & 7.1 \\
\hline Fabricated metal products & & -8.6 & 4.9 & 5.6 & 0.7 & 2.1 & 17.8 & 9.6 \\
\hline
\end{tabular}

Source: Data provided by the Bangladesh Bureau of Statistics.

1/ Excluding mining and electricity. 
Table 5. Bangladesh: Energy Statistics, 1999/00-2005/06

\begin{tabular}{|c|c|c|c|c|c|c|c|}
\hline & $1999 / 00$ & $2000 / 01$ & $2001 / 02$ & $2002 / 03$ & $2003 / 04$ & $2004 / 05$ & $2005 / 06$ \\
\hline & \multicolumn{7}{|c|}{ (In thousands of metric tons) } \\
\hline \multicolumn{8}{|l|}{ Petroleum products } \\
\hline Imports & 2,206 & 2,265 & 2,465 & 2,554 & 2,312 & 2,656 & 2,294 \\
\hline Domestically refined & 1,340 & 1,319 & 1,350 & 1,323 & 1,347 & 1,226 & 1,405 \\
\hline Consumption & 3,487 & 3,572 & 3,715 & 3,769 & 3,570 & 3,782 & 3,956 \\
\hline Exports and change in stocks & 59 & 12 & 100 & 108 & 89 & 80 & 115 \\
\hline & \multicolumn{7}{|c|}{ (In millions of cubic feet) } \\
\hline \multicolumn{8}{|l|}{ Natural gas } \\
\hline Production & 332,389 & 373,372 & 391,530 & 245,228 & 452,774 & 486,756 & 526,925 \\
\hline Consumption & 310,054 & 348,772 & 364,651 & 231,947 & 427,670 & 456,181 & 495,142 \\
\hline Power & 149,255 & 175,204 & 190,038 & 109,333 & 199,401 & 211,023 & 224,394 \\
\hline Fertilizer & 84,894 & 88,464 & 78,785 & 57,880 & 92,804 & 93,986 & 89,086 \\
\hline Industry & 41,372 & 48,096 & 53,554 & 36,162 & 46,489 & 51,417 & 63,261 \\
\hline Commercial & 4,857 & 5,136 & 5,518 & 3,323 & 4,829 & 4,862 & 5,208 \\
\hline Domestic & 29,676 & 31,872 & 36,756 & 25,249 & 49,222 & 52,669 & 56,747 \\
\hline Others $1 /$ & & & & & & 42,224 & 56,446 \\
\hline System losses & 22,335 & 24,600 & 26,879 & 13,281 & 25,104 & 30,576 & 31,784 \\
\hline & \multicolumn{7}{|c|}{ (In millions of kilowatt hours) } \\
\hline \multicolumn{8}{|l|}{ Electricity } \\
\hline Generation & 14,450 & 15,563 & 17,021 & 12,881 & 13,342 & 14,067 & 1,542 \\
\hline \multicolumn{8}{|l|}{ Of which: } \\
\hline Based on natural gas & 12,603 & 13,266 & 13,302 & 11,331 & 11,548 & 12,171 & 13,384 \\
\hline System losses (net) & 2,270 & 2,252 & 2,207 & 2,091 & 2,038 & 1,967 & 1,788 \\
\hline
\end{tabular}

Sources: Data provided by the Bangladesh authorities; BPDB; BOGMC; and BPC.

1/ CNG, Tea-estate and CAP. 
Table 6. Bangladesh: Retail Prices of Petroleum Products, 1999/00-2006/07 1/

\begin{tabular}{|c|c|c|c|c|c|c|c|c|}
\hline & $1999 / 00$ & $2000 / 01$ & $2001 / 02$ & $2002 / 03$ & $2003 / 04$ & $2004 / 05$ & $2005 / 06$ & April 2007 \\
\hline & \multicolumn{8}{|c|}{ (Taka/liter) } \\
\hline Premium gasoline & 23.00 & 25.00 & 30.00 & 35.00 & 35.00 & 35.00 & 58.00 & 67.00 \\
\hline Regular gasoline & 21.00 & 23.00 & 28.00 & 33.00 & 33.00 & 35.00 & 56.00 & 65.00 \\
\hline Kerosene & 12.95 & 15.50 & 17.00 & 17.00 & 20.00 & 25.00 & 33.00 & 40.00 \\
\hline Diesel oil & 12.95 & 15.50 & 17.00 & 20.00 & 20.00 & 26.00 & 33.00 & 40.00 \\
\hline Fuel oil & 5.00 & 6.50 & 12.50 & 10.00 & 12.00 & 12.00 & 20.00 & 20.00 \\
\hline \multirow[t]{2}{*}{ Jet oil } & 16.62 & 19.05 & 21.15 & 24.00 & 27.00 & 41.75 & 56.00 & 56.00 \\
\hline & \multicolumn{8}{|c|}{ (U.S. dollar/gallon) } \\
\hline Premium gasoline & 1.71 & 1.66 & 1.96 & 2.29 & 2.20 & 2.08 & 3.15 & 3.68 \\
\hline Regular gasoline & 1.56 & 1.53 & 1.83 & 2.16 & 2.07 & 2.08 & 3.04 & 3.57 \\
\hline Kerosene & 0.96 & 1.03 & 1.11 & 1.11 & 1.25 & 1.48 & 1.79 & 2.20 \\
\hline Diesel oil & 0.96 & 1.03 & 1.11 & 1.31 & 1.25 & 1.54 & 1.79 & 2.20 \\
\hline Fuel oil & 0.37 & 0.43 & 0.82 & 0.65 & 0.75 & 0.71 & 1.09 & 1.10 \\
\hline Jet oil & 1.23 & 1.27 & 1.38 & 1.57 & 1.69 & 2.48 & 3.04 & 3.07 \\
\hline \multicolumn{9}{|l|}{ Memorandum items: } \\
\hline Average crude price (US\$/barrel) & 29.62 & 26.97 & 24.49 & 27.91 & 35.54 & 53.89 & 68.29 & 65.10 \\
\hline Conversion: Taka/US\$ & 51.00 & 57.00 & 57.90 & 57.90 & 60.35 & 63.75 & 69.73 & 68.94 \\
\hline
\end{tabular}

Source: Bangladesh Petroleum Corporation.

1/ End-of-period. 
Table 7. Bangladesh: Consumer Price Index, National, 1999/00-2004/05

(Based on 1995/96 weights; percentage change)

\begin{tabular}{|c|c|c|c|c|c|c|c|c|c|c|}
\hline \multirow[b]{2}{*}{ Period } & \multirow[b]{2}{*}{ General } & \multirow[b]{2}{*}{$\begin{array}{r}\text { Food } \\
\text { Beverages } \\
\text { and } \\
\text { Tobacco }\end{array}$} & \multirow[b]{2}{*}{$\begin{array}{r}\text { Total } \\
\text { Nonfood }\end{array}$} & \multicolumn{7}{|c|}{ Nonfood } \\
\hline & & & & $\begin{array}{r}\text { Clothing } \\
\text { and } \\
\text { footwear }\end{array}$ & $\begin{array}{r}\text { Gross rent } \\
\text { fuel and } \\
\text { lighting }\end{array}$ & $\begin{array}{r}\text { Furniture } \\
\text { furnishing } \\
\text { household } \\
\text { equipments } \\
\text { and } \\
\text { operation }\end{array}$ & $\begin{array}{r}\text { Medical } \\
\text { care and } \\
\text { health } \\
\text { expenses }\end{array}$ & $\begin{array}{r}\text { Transport } \\
\text { and } \\
\text { communi- } \\
\text { cations }\end{array}$ & $\begin{array}{r}\text { Recreation } \\
\text { enter- } \\
\text { tainment, } \\
\text { education, } \\
\text { and cultural } \\
\text { services }\end{array}$ & $\begin{array}{r}\text { Misc. } \\
\text { goods and } \\
\text { services }\end{array}$ \\
\hline \multirow[t]{2}{*}{ Weights } & 100.0 & 58.8 & 41.2 & 6.9 & 16.9 & 2.7 & 2.8 & 4.2 & 4.1 & 3.6 \\
\hline & \multicolumn{10}{|c|}{ (Annual average percentage changes) } \\
\hline $2001 / 02$ & 2.8 & 1.6 & 4.6 & 2.2 & 4.6 & 4.3 & 4.9 & 6.2 & 7.7 & 3.5 \\
\hline 2002/03 & 4.4 & 3.5 & 5.7 & 4.8 & 5.0 & 5.0 & 6.6 & 10.5 & 4.5 & 4.8 \\
\hline 2003/04 & 5.8 & 6.9 & 4.4 & 4.4 & 3.8 & 4.2 & 6.3 & 7.1 & 3.2 & 3.4 \\
\hline $2004 / 05$ & 6.5 & 7.9 & 4.3 & 4.3 & 3.8 & 3.8 & 5.3 & 5.4 & 6.3 & 2.8 \\
\hline \multirow[t]{2}{*}{$2005 / 06$} & 7.2 & 7.8 & 6.4 & 4.4 & 7.5 & 5.6 & 4.4 & 6.5 & 10.1 & 4.1 \\
\hline & \multicolumn{10}{|c|}{ (End of period annual percentage changes) } \\
\hline \multicolumn{11}{|l|}{$2005 / 06$} \\
\hline July & 7.7 & 9.2 & 5.5 & 5.6 & 5.2 & 5.2 & 3.6 & 6.3 & 9.5 & 2.8 \\
\hline August & 7.9 & 9.4 & 5.7 & 5.5 & 5.7 & 5.4 & 3.9 & 5.5 & 10.5 & 4.8 \\
\hline September & 7.0 & 7.2 & 6.9 & 5.4 & 7.8 & 6.1 & 4.1 & 6.5 & 12.4 & 3.4 \\
\hline October & 7.3 & 7.4 & 7.1 & 5.0 & 8.5 & 5.8 & 4.6 & 7.4 & 10.8 & 3.9 \\
\hline November & 8.0 & 8.6 & 7.0 & 4.8 & 8.4 & 5.4 & 4.6 & 7.4 & 10.8 & 3.8 \\
\hline December & 7.1 & 7.1 & 7.1 & 4.7 & 8.8 & 5.5 & 4.8 & 7.3 & 10.6 & 3.6 \\
\hline January & 6.6 & 6.7 & 6.7 & 4.4 & 8.1 & 5.4 & 4.5 & 7.0 & 10.3 & 3.7 \\
\hline February & 5.7 & 5.5 & 6.3 & 4.2 & 7.1 & 5.5 & 4.3 & 7.1 & 9.6 & 4.3 \\
\hline March & 6.2 & 6.1 & 6.6 & 3.9 & 7.8 & 6.0 & 4.7 & 7.1 & 9.9 & 4.9 \\
\hline April & 7.5 & 8.3 & 6.4 & 3.6 & 7.8 & 5.6 & 4.8 & 6.3 & 9.6 & 4.9 \\
\hline May & 7.6 & 8.9 & 5.9 & 2.8 & 7.3 & 5.7 & 4.6 & 5.1 & 9.0 & 4.8 \\
\hline June & 7.5 & 8.8 & 5.7 & 2.5 & 7.2 & 5.8 & 4.2 & 5.5 & 8.3 & 4.4 \\
\hline \multicolumn{11}{|l|}{$2006 / 07$} \\
\hline July & 6.8 & 7.4 & 5.9 & 3.2 & 7.5 & 5.6 & 4.6 & 5.3 & 8.0 & 4.3 \\
\hline August & 6.7 & 7.4 & 5.6 & 3.4 & 6.9 & 5.9 & 4.8 & 5.4 & 6.6 & 4.2 \\
\hline September & 6.9 & 8.2 & 4.9 & 4.8 & 4.9 & 5.7 & 4.8 & 4.8 & 4.0 & 5.1 \\
\hline October & 7.3 & 9.0 & 4.8 & 5.4 & 5.1 & 5.7 & 4.7 & 3.9 & 3.3 & 4.5 \\
\hline November & 6.4 & 7.3 & 4.9 & 5.3 & 5.3 & 5.7 & 5.0 & 4.1 & 3.2 & 4.5 \\
\hline December & 6.1 & 7.1 & 4.6 & 5.1 & 4.8 & 5.8 & 4.9 & 4.0 & 3.2 & 4.6 \\
\hline January & 5.9 & 6.6 & 5.0 & 5.4 & 5.3 & 6.6 & 5.1 & 4.1 & 3.2 & 4.9 \\
\hline February & 7.3 & 8.4 & 5.7 & 6.1 & 6.5 & 5.0 & 6.0 & 4.5 & 3.2 & 5.3 \\
\hline March & 7.4 & 8.5 & 5.8 & 6.8 & 6.3 & 8.5 & 5.6 & 4.1 & 2.7 & 5.3 \\
\hline
\end{tabular}

Source: Bangladesh Bureau of Statistics. 
Table 8. Bangladesh: Labor Market Statistics, 1990/91-2005/06 1/

\begin{tabular}{|c|c|c|c|c|c|c|c|c|c|c|}
\hline & $1990 / 91$ & 1995/96 & $1998 / 99$ & 1999/00 & $2000 / 01$ & $2001 / 02$ & $2002 / 03$ & 2003/04 & $2004 / 05$ & $2005 / 06$ \\
\hline Total population (in millions) & 111.5 & 122.0 & 126.0 & 127.5 & 129.2 & 131.6 & 133.4 & 135.2 & 137.0 & 138.8 \\
\hline Male & 57.3 & 63.0 & $\ldots$ & 66.6 & 65.8 & $\ldots$ & 68.3 & $\ldots$ & $\ldots$ & $\ldots$ \\
\hline Female & 54.2 & 59.0 & $\ldots$ & 60.9 & 63.4 & $\ldots$ & 65.1 & $\ldots$ & $\ldots$ & $\ldots$ \\
\hline Population growth rate (in percent) $2 /$ & 1.9 & 2.0 & 2.0 & 1.5 & 1.5 & 1.5 & 1.5 & $\ldots$ & $\ldots$ & $\ldots$ \\
\hline Total labor force (in millions) $3 /$ & 51.0 & 56.0 & $\ldots$ & 40.7 & $\ldots$ & $\ldots$ & 46.3 & $\ldots$ & $\ldots$ & $\ldots$ \\
\hline Male & 31.0 & 35.0 & $\ldots$ & 32.2 & $\ldots$ & $\ldots$ & 36.0 & $\ldots$ & $\ldots$ & $\ldots$ \\
\hline Female & 20.0 & 21.0 & $\ldots$ & 8.5 & $\ldots$ & $\ldots$ & 10.3 & $\ldots$ & $\ldots$ & $\ldots$ \\
\hline Types of employment (in percent) & 100.0 & 100.0 & $\ldots$ & 100.0 & $\ldots$ & $\ldots$ & 100.0 & $\ldots$ & $\ldots$ & $\ldots$ \\
\hline Formal & 11.7 & 12.4 & $\ldots$ & 24.8 & $\ldots$ & $\ldots$ & 20.9 & $\ldots$ & $\ldots$ & $\ldots$ \\
\hline Nonformal & 87.9 & 87.6 & $\ldots$ & 75.2 & $\ldots$ & $\ldots$ & 79.1 & $\ldots$ & $\ldots$ & $\ldots$ \\
\hline Family-based & 47.2 & 40.1 & $\ldots$ & 4.3 & $\ldots$ & $\ldots$ & 14.4 & $\ldots$ & $\ldots$ & $\ldots$ \\
\hline Daily basis & 13.9 & 17.9 & $\ldots$ & 24.3 & $\ldots$ & $\ldots$ & 19.9 & $\ldots$ & $\ldots$ & $\ldots$ \\
\hline Self-employed & 26.8 & 29.6 & $\ldots$ & 46.6 & $\ldots$ & $\ldots$ & 44.8 & $\cdots$ & $\ldots$ & $\ldots$ \\
\hline Employment by activity (in percent) & 100.0 & 100.0 & $\ldots$ & 100.0 & $\ldots$ & $\ldots$ & 100.0 & $\ldots$ & $\ldots$ & $\ldots$ \\
\hline Agriculture, forest, fisheries & 66.4 & 63.2 & $\ldots$ & 50.8 & $\ldots$ & $\ldots$ & 51.7 & $\ldots$ & $\ldots$ & $\ldots$ \\
\hline Mining and quarrying & $\ldots$ & $\ldots$ & $\ldots$ & 0.5 & $\ldots$ & $\ldots$ & 0.2 & $\ldots$ & $\ldots$ & $\ldots$ \\
\hline Manufacturing & 11.8 & 7.5 & $\ldots$ & 9.6 & $\ldots$ & $\ldots$ & 9.8 & $\ldots$ & $\ldots$ & $\ldots$ \\
\hline Electricity, gas, and water & 0.1 & 0.2 & $\ldots$ & 0.4 & $\ldots$ & $\ldots$ & 0.2 & $\ldots$ & $\ldots$ & $\ldots$ \\
\hline Construction & 1.0 & 1.8 & $\ldots$ & 2.8 & $\ldots$ & $\ldots$ & 3.5 & $\ldots$ & $\ldots$ & $\ldots$ \\
\hline Trade services & 8.5 & 11.2 & $\ldots$ & 15.8 & $\ldots$ & $\ldots$ & 15.1 & $\ldots$ & $\ldots$ & $\ldots$ \\
\hline Transport and communication & 3.2 & 4.2 & $\ldots$ & 6.3 & $\ldots$ & $\ldots$ & 6.8 & $\ldots$ & $\ldots$ & $\ldots$ \\
\hline Finance and business service & 0.6 & 0.4 & $\ldots$ & 1.0 & $\ldots$ & $\ldots$ & 0.9 & $\ldots$ & $\ldots$ & $\ldots$ \\
\hline Community and personal service & 3.8 & 9.3 & $\ldots$ & 7.5 & $\ldots$ & $\ldots$ & 5.8 & $\ldots$ & $\ldots$ & $\ldots$ \\
\hline Others & 4.5 & 2.2 & $\ldots$ & 5.5 & $\ldots$ & $\ldots$ & 6.0 & $\ldots$ & $\ldots$ & $\ldots$ \\
\hline Wage rate index (general) $(1969-70=100)$ & 1,482 & 1,900 & 2,259 & 2,390 & 2,489 & 2,637 & 2,926 & 3,111 & 3,293 & 3,507 \\
\hline Agriculture & 1,321 & 1,738 & 1,950 & 2,037 & 2,141 & 2,262 & 2,443 & 2,582 & 2,719 & 2,926 \\
\hline Manufacturing & 1,575 & 2,064 & 2,522 & 2,701 & 2,832 & 3,035 & 3,501 & 3,765 & 4,015 & 4,293 \\
\hline Construction & 1,487 & 1,754 & 2,163 & 2,286 & 2,356 & 2,444 & 2,624 & 2,669 & 2,758 & 2,889 \\
\hline CPI for industrial workers $(1969-70=100)$ & 1,386 & 1,674 & 1,921 & 1,973 & 1,999 & 2,024 & 2,068 & 2,129 & 2,216 & 2,350 \\
\hline
\end{tabular}

Source: Bangladesh Bureau of Statistics.

$1 /$ Based on labor force surveys.

2/ Natural growth rate (crude birth minus crude death).

3/ Aged 10 years and above. 
Table 9. Bangladesh: Central Government Operations, 2000/01-2005/06 1/

\begin{tabular}{|c|c|c|c|c|c|c|}
\hline & $2000 / 01$ & $2001 / 02$ & $2002 / 03$ & $2003 / 04$ & $2004 / 05$ & $2005 / 06$ \\
\hline & \multicolumn{6}{|c|}{ (In billions of taka) } \\
\hline Total revenue & 228.4 & 276.8 & 309.7 & 339.0 & 389.2 & 443.7 \\
\hline Tax revenue & 192.6 & 210.3 & 248.2 & 274.3 & 314.1 & 354.2 \\
\hline NBR taxes & 182.6 & 199.2 & 237.6 & 261.9 & 299.9 & 338.9 \\
\hline VAT, supplementary duties, excises & 99.5 & 111.0 & 124.8 & 141.0 & 161.6 & 182.9 \\
\hline Customs duties & 49.6 & 51.7 & 66.9 & 70.9 & 97.6 & 78.4 \\
\hline Taxes on income and profits & 31.7 & 35.2 & 43.7 & 47.1 & 56.7 & 71.5 \\
\hline Other NBR taxes & 1.8 & 1.3 & 2.2 & 2.9 & 2.6 & 6.2 \\
\hline Non-NBR taxes & 10.0 & 11.1 & 10.7 & 12.4 & 14.2 & 15.3 \\
\hline Nontax revenue & 35.8 & 66.5 & 61.5 & 64.7 & 75.1 & 89.5 \\
\hline Total expenditure & 374.5 & 389.9 & 411.4 & 441.6 & 513.3 & 578.1 \\
\hline Current expenditure & 194.4 & 218.2 & 244.5 & 258.6 & 312.5 & 350.4 \\
\hline Pay and allowances & 58.8 & 68.8 & 71.2 & 76.6 & 84.2 & 100.4 \\
\hline Goods and services & 26.8 & 33.2 & 42.5 & 45.1 & 56.4 & 60.7 \\
\hline Interest payments & 41.3 & 49.3 & 56.6 & 54.5 & 61.8 & 75.1 \\
\hline Subsidies and transfers & 57.4 & 58.4 & 70.2 & 78.9 & 103.2 & 108.6 \\
\hline Block allocations & 10.1 & 8.4 & 4.0 & 3.6 & 6.9 & 5.7 \\
\hline Food account surplus(-)/deficit(+) & 2.1 & 2.1 & -2.7 & 3.5 & 0.6 & -1.2 \\
\hline Annual Development Program (ADP) & 165.8 & 152.3 & 163.0 & 167.9 & 185.8 & 194.7 \\
\hline Non-ADP capital and net lending & 12.0 & 17.2 & 6.6 & 16.0 & 24.2 & 41.3 \\
\hline Extraordinary expenditures & 0.0 & 0.0 & 12.3 & 7.8 & 1.9 & 0.0 \\
\hline Check float plus discrepancy & -16.0 & 14.2 & -12.2 & -12.2 & -11.8 & -7.0 \\
\hline Overall balance (excluding grants) & -130.0 & -127.4 & -101.7 & -102.6 & -124.0 & -134.5 \\
\hline Primary balance (excluding grants) & -88.7 & -78.0 & -45.1 & -48.1 & -62.2 & -59.4 \\
\hline Net financing & 130.0 & 127.4 & 101.7 & 102.6 & 124.0 & 134.5 \\
\hline External & 50.2 & 58.6 & 64.2 & 42.8 & 60.2 & 48.1 \\
\hline Domestic & 78.9 & 68.1 & 37.5 & 59.8 & 63.8 & 86.4 \\
\hline Bank & 36.8 & 21.7 & -10.7 & 13.4 & 35.0 & 58.8 \\
\hline \multirow[t]{2}{*}{ Nonbank } & 42.1 & 46.4 & 48.2 & 46.4 & 28.8 & 27.6 \\
\hline & \multicolumn{6}{|c|}{ (In percent of GDP) } \\
\hline Total revenue & 9.0 & 10.1 & 10.3 & 10.2 & 10.5 & 10.7 \\
\hline Tax revenue & 7.6 & 7.7 & 8.3 & 8.2 & 8.5 & 8.5 \\
\hline NBR taxes & 7.2 & 7.3 & 7.9 & 7.9 & 8.1 & 8.1 \\
\hline Non-NBR taxes & 0.4 & 0.4 & 0.4 & 0.4 & 0.4 & 0.4 \\
\hline Nontax revenue & 1.4 & 2.4 & 2.0 & 1.9 & 2.0 & 2.1 \\
\hline Total expenditure & 14.8 & 14.3 & 13.7 & 13.3 & 13.8 & 13.9 \\
\hline Current expenditure & 7.7 & 8.0 & 8.1 & 7.8 & 8.4 & 8.4 \\
\hline Pay and allowances & 2.3 & 2.5 & 2.4 & 2.3 & 2.3 & 2.4 \\
\hline Goods and services & 1.1 & 1.2 & 1.4 & 1.4 & 1.5 & 1.5 \\
\hline Interest & 1.6 & 1.8 & 1.9 & 1.6 & 1.7 & 1.8 \\
\hline Subsidies and transfers & 2.3 & 2.1 & 2.3 & 2.4 & 2.8 & 2.6 \\
\hline Block allocations & 0.4 & 0.3 & 0.1 & 0.1 & 0.2 & 0.1 \\
\hline Annual Development Program & 6.5 & 5.6 & 5.4 & 5.0 & 5.0 & 4.7 \\
\hline Non-ADP capital and net lending & 0.5 & 0.6 & 0.2 & 0.5 & 0.7 & 1.0 \\
\hline Other expenditures & 0.0 & 0.0 & 0.4 & 0.2 & 0.1 & 0.0 \\
\hline Check float plus discrepancy & -0.6 & 0.5 & -0.4 & -0.4 & -0.3 & -0.2 \\
\hline Overall balance (excluding grants) & -5.1 & -4.7 & -3.4 & -3.1 & -3.3 & -3.2 \\
\hline Primary balance (excluding grants) & -3.5 & -2.9 & -1.5 & -1.4 & -1.7 & -1.4 \\
\hline Net financing & 5.1 & 4.7 & 3.4 & 3.1 & 3.3 & 3.2 \\
\hline External financing (net) & 2.0 & 2.1 & 2.1 & 1.3 & 1.6 & 1.2 \\
\hline Domestic financing & 3.1 & 2.5 & 1.2 & 1.8 & 1.7 & 2.1 \\
\hline Banks & 1.5 & 0.8 & -0.4 & 0.4 & 0.9 & 1.4 \\
\hline Nonbank & 1.7 & 1.7 & 1.6 & 1.4 & 0.8 & 0.7 \\
\hline \multicolumn{7}{|l|}{ Memorandum item: } \\
\hline Public debt & 50.8 & 52.9 & 51.1 & 48.7 & 47.5 & 46.8 \\
\hline External & 33.5 & 34.2 & 32.8 & 30.4 & 29.3 & 28.6 \\
\hline Domestic & 17.3 & 18.7 & 18.3 & 18.3 & 18.1 & 18.2 \\
\hline
\end{tabular}

Sources: Data provided by the Bangladesh authorities; and IMF staff estimates.

1/ Fiscal year ends June 30 . 
Table 10. Bangladesh: Central Government Revenue, 2000/01-2005/06 1/

\begin{tabular}{|c|c|c|c|c|c|c|}
\hline & $2000 / 01$ & $2001 / 02$ & $2002 / 03$ & $2003 / 04$ & $2004 / 05$ & $2005 / 06$ \\
\hline & \multicolumn{6}{|c|}{ (In billions of taka) } \\
\hline Total revenue & 228.4 & 276.8 & 309.7 & 339.0 & 389.2 & 443.7 \\
\hline Tax revenue & 192.6 & 210.3 & 248.2 & 274.3 & 314.1 & 354.2 \\
\hline NBR taxes & 182.6 & 199.2 & 237.6 & 261.9 & 299.9 & 338.9 \\
\hline VAT, supplementary duties, excises & 99.5 & 111.0 & 124.8 & 141.0 & 161.6 & 182.9 \\
\hline VAT & 63.1 & 70.4 & 77.4 & 87.1 & 104.6 & 121.7 \\
\hline Import & 37.5 & 38.5 & 41.1 & 44.0 & 53.5 & 58.5 \\
\hline Domestic & 25.6 & 32.0 & 36.2 & 43.2 & 51.1 & 63.2 \\
\hline Supplementary duties & 33.7 & 37.7 & 44.2 & 52.3 & 55.6 & 59.6 \\
\hline Import & 12.7 & 13.7 & 12.7 & 16.9 & 18.5 & 15.7 \\
\hline Domestic & 21.0 & 24.0 & 31.5 & 35.5 & 37.0 & 43.9 \\
\hline Excises & 2.8 & 2.9 & 3.2 & 1.6 & 1.4 & 1.5 \\
\hline Customs duties & 49.6 & 51.7 & 66.9 & 70.9 & 79.1 & 78.4 \\
\hline Taxes on income and profits & 31.7 & 35.2 & 43.7 & 47.1 & 56.7 & 71.5 \\
\hline Other NBR taxes & 1.8 & 1.3 & 2.2 & 2.9 & 2.6 & 6.2 \\
\hline Non-NBR taxes & 10.0 & 11.1 & 10.7 & 12.4 & 14.2 & 15.3 \\
\hline \multirow[t]{2}{*}{ Nontax revenue } & 35.8 & 66.5 & 61.5 & 64.7 & 75.1 & 89.5 \\
\hline & \multicolumn{6}{|c|}{ (In percent of GDP) } \\
\hline Total revenue & 9.0 & 10.1 & 10.3 & 10.2 & 10.5 & 10.7 \\
\hline Tax revenue & 7.6 & 7.7 & 8.3 & 8.2 & 8.5 & 8.5 \\
\hline NBR taxes & 7.2 & 7.3 & 7.9 & 7.9 & 8.1 & 8.1 \\
\hline VAT, supplementary duties, excises & 3.9 & 4.1 & 4.2 & 4.2 & 4.4 & 4.4 \\
\hline VAT & 2.5 & 2.6 & 2.6 & 2.6 & 2.8 & 2.9 \\
\hline Import & 1.5 & 1.4 & 1.4 & 1.3 & 1.4 & 1.4 \\
\hline Domestic & 1.0 & 1.2 & 1.2 & 1.3 & 1.4 & 1.5 \\
\hline Supplementary duties & 1.3 & 1.4 & 1.5 & 1.6 & 1.5 & 1.4 \\
\hline Import & 0.5 & 0.5 & 0.4 & 0.5 & 0.5 & 0.4 \\
\hline Domestic & 0.8 & 0.9 & 1.0 & 1.1 & 1.0 & 1.1 \\
\hline Excises & 0.1 & 0.1 & 0.1 & 0.0 & 0.0 & 0.0 \\
\hline Customs duties & 2.0 & 1.9 & 2.2 & 2.1 & 2.1 & 1.9 \\
\hline Taxes on income and profits & 1.2 & 1.3 & 1.5 & 1.4 & 1.5 & 1.7 \\
\hline Other NBR taxes & 0.1 & 0.0 & 0.1 & 0.1 & 0.1 & 0.1 \\
\hline Non-NBR taxes & 0.4 & 0.4 & 0.4 & 0.4 & 0.4 & 0.4 \\
\hline Nontax revenue & 1.4 & 2.4 & 2.0 & 1.9 & 2.0 & 2.1 \\
\hline
\end{tabular}

Sources: Data provided by the Bangladesh authorities; and IMF staff estimates.

$1 /$ Fiscal year ends June 30 . 
Table 11. Bangladesh: Central Government Current Expenditure, 2000/01-2005/06 1/

\begin{tabular}{|c|c|c|c|c|c|c|}
\hline & $2000 / 01$ & $2001 / 02$ & $2002 / 03$ & $2003 / 04$ & $2004 / 05$ & $2005 / 06$ \\
\hline & \multicolumn{6}{|c|}{ (In billions of taka) } \\
\hline Current expenditure & 194.4 & 218.2 & 244.5 & 258.6 & 312.5 & 350.4 \\
\hline Pay and allowances & 58.8 & 68.8 & 71.2 & 76.6 & 84.2 & 100.4 \\
\hline Goods and services & 26.8 & 33.2 & 42.5 & 45.1 & 56.4 & 60.7 \\
\hline Interest payments & 41.3 & 49.3 & 56.6 & 54.5 & 61.8 & 75.1 \\
\hline Domestic & 32.6 & 41.5 & 46.9 & 45.5 & 47.8 & 62.6 \\
\hline Foreign & 8.7 & 7.8 & 9.7 & 9.1 & 14.0 & 12.5 \\
\hline Subsidies and transfers & 57.4 & 58.4 & 70.2 & 78.9 & 103.2 & 108.6 \\
\hline Subsidies & 0.0 & 0.0 & 13.5 & 11.9 & 21.6 & 16.9 \\
\hline Transfers & 0.0 & 0.0 & 56.7 & 67.0 & 81.6 & 91.7 \\
\hline \multirow[t]{2}{*}{ Block allocations } & 10.1 & 8.4 & 4.0 & 3.6 & 6.9 & 5.7 \\
\hline & \multicolumn{6}{|c|}{ (In percent of GDP) } \\
\hline Current expenditure & 7.7 & 8.0 & 8.1 & 7.8 & 8.4 & 8.4 \\
\hline Pay and allowances & 2.3 & 2.5 & 2.4 & 2.3 & 2.3 & 2.4 \\
\hline Goods and services & 1.1 & 1.2 & 1.4 & 1.4 & 1.5 & 1.5 \\
\hline Interest payments & 1.6 & 1.8 & 1.9 & 1.6 & 1.7 & 1.8 \\
\hline Domestic & 1.3 & 1.5 & 1.6 & 1.4 & 1.3 & 1.5 \\
\hline Foreign & 0.3 & 0.3 & 0.3 & 0.3 & 0.4 & 0.3 \\
\hline Subsidies and transfers & 2.3 & 2.1 & 2.3 & 2.4 & 2.8 & 2.6 \\
\hline Subsidies & 0.0 & 0.0 & 0.4 & 0.4 & 0.6 & 0.4 \\
\hline Transfers & 0.0 & 0.0 & 1.9 & 2.0 & 2.2 & 2.2 \\
\hline Block allocations & 0.4 & 0.3 & 0.1 & 0.1 & 0.2 & 0.1 \\
\hline
\end{tabular}

Sources: Data provided by the Bangladesh authorities; and IMF staff estimates. 
Table 12. Bangladesh: Central Government Food Account, 1999/00-2005/06

(In billions of taka)

\begin{tabular}{lrrrrrrr}
\hline & $1999 / 00$ & $2000 / 01$ & $2001 / 02$ & $2002 / 03$ & $2003 / 04$ & $2004 / 05$ & $2005 / 06$ \\
\hline Expenditure & 25.7 & 21.4 & 19.7 & 15.9 & 18.6 & 24.1 & 23.3 \\
Imports & 8.8 & 5.4 & 5.5 & 2.9 & 3.7 & 5.4 & 4.1 \\
$\quad$ Grants and loans & 8.4 & 5.4 & 5.4 & 2.9 & 3.5 & 3.8 & 2.9 \\
$\quad$ Commercial (cash) & 0.4 & 0.0 & 0.0 & 0.0 & 0.3 & 1.5 & 1.2 \\
Domestic procurement & 13.5 & 13.1 & 11.4 & 10.2 & 12.2 & 15.9 & 16.2 \\
External freight & 0.2 & 0.1 & 0.3 & 0.3 & 0.3 & 0.3 & 0.2 \\
Other expenses 1/ & 3.2 & 2.7 & 2.6 & 2.4 & 2.3 & 2.5 & 2.7 \\
Receipts & 22.1 & 21.9 & 17.6 & 15.0 & 14.0 & 17.0 & 17.1 \\
Gross sales & 1.2 & 1.1 & 0.8 & 3.9 & 0.9 & 4.0 & 1.2 \\
Food-for-education 2/ & 3.8 & 4.1 & 2.8 & 0.0 & 0.0 & 0.0 & 0.0 \\
Nonmonetized distribution $3 /$ & 17.0 & 16.6 & 14.0 & 11.1 & 13.1 & 13.0 & 15.9 \\
$\quad$ Food-for-work & 9.6 & 8.0 & 7.0 & 4.3 & 3.0 & 2.1 & 3.9 \\
$\quad$ Vulnerable groups, etc. & 7.4 & 8.7 & 7.0 & 6.8 & 10.1 & 10.9 & 12.1 \\
& & & & & & -7.9 & -6.1 \\
Food account balance & -3.7 & 0.5 & -2.1 & -0.9 & -4.6 & -7.1 & \\
\hline
\end{tabular}

Sources: Ministry of Finance; and Ministry of Food.

1/ Includes establishment costs, other operating expenses, and reconciliation differences.

2/ Food-based social protection programs are monetized starting FY03.

$3 /$ This item represents the valuation of nonmonetized distribution. In effect, it represents transfers to the food account from other expenditure categories. 
Table 13. Bangladesh: Nonfinancial Public Corporations Operations, 2000/01-2005/06

(In billions of taka)

\begin{tabular}{|c|c|c|c|c|c|c|}
\hline & $2000 / 01$ & $2001 / 02$ & $2002 / 03$ & $2003 / 04$ & $2004 / 05$ & $\begin{array}{r}\text { Est. } \\
2005 / 06\end{array}$ \\
\hline Operating revenue $1 /$ & 255.9 & 274.6 & 305.0 & 325.4 & 362.7 & 450.8 \\
\hline Operating expenditure & 273.7 & 279.7 & 301.3 & 324.5 & 387.5 & 490.9 \\
\hline Wages and salaries & 18.3 & 18.1 & 17.8 & 17.8 & 16.6 & 20.8 \\
\hline Purchase of goods and services & 234.1 & 240.1 & 268.1 & 287.0 & 353.2 & 451.9 \\
\hline Depreciation & 21.2 & 21.5 & 15.3 & 19.7 & 17.6 & 18.1 \\
\hline Operating Surplus & -17.7 & -5.1 & 3.8 & 0.9 & -24.7 & -40.1 \\
\hline Nonoperating income & 5.7 & 4.5 & 11.5 & 6.8 & 5.9 & 6.8 \\
\hline Interest payments & 12.3 & 11.4 & 11.5 & 9.6 & 9.0 & 10.8 \\
\hline Income before taxes & -24.3 & -12.0 & 3.8 & -1.9 & -27.9 & -44.1 \\
\hline Profit distributions & 1.8 & 2.0 & 2.2 & 2.6 & 0.6 & 1.3 \\
\hline Dividends & 1.6 & 1.8 & 1.9 & 2.2 & 0.6 & 1.2 \\
\hline Profit sharing & 0.2 & 0.2 & 0.3 & 0.4 & 0.1 & 0.1 \\
\hline Income tax & 1.8 & 2.1 & 2.6 & 3.4 & 1.3 & 1.4 \\
\hline After tax retained income & -27.9 & -16.0 & -0.9 & -7.9 & -29.8 & -46.7 \\
\hline Gross savings $2 /$ & -6.7 & 5.4 & 14.5 & 11.8 & -12.2 & -28.6 \\
\hline Gross fixed capital formation & 40.6 & 38.5 & 54.5 & 44.5 & 49.4 & 72.5 \\
\hline Financing Requirements & 47.3 & 33.0 & 40.0 & 32.8 & 61.6 & 101.1 \\
\hline Net cash borrowing & 9.0 & 11.1 & 31.6 & 7.4 & 15.9 & 17.5 \\
\hline Drawings & 26.8 & 39.4 & 51.3 & 32.1 & 32.6 & 33.4 \\
\hline Repayments & -17.8 & -28.3 & -19.7 & -24.7 & -16.7 & -15.9 \\
\hline Equity Injection & 25.1 & 9.6 & 16.6 & 25.8 & 19.7 & 21.5 \\
\hline Finance deficit $3 /$ & 13.2 & 12.3 & -8.2 & -0.5 & 25.9 & 62.2 \\
\hline \multicolumn{7}{|l|}{ Memorandum items: } \\
\hline Total Assets & 897.2 & 968.5 & 1130.7 & 1292.1 & 1243.5 & 1337.0 \\
\hline Equity & 224.9 & 250.7 & 334.0 & 508.1 & 364.1 & 333.3 \\
\hline Debt & 672.3 & 717.8 & 796.7 & 783.9 & 879.4 & 1003.7 \\
\hline Employment (thousands) & 244.7 & 214.7 & 207.5 & 195.1 & 186.4 & 183.6 \\
\hline \multicolumn{7}{|l|}{ Profitability (in percent) } \\
\hline Operating surplus/operating revenue & -6.9 & -1.8 & 1.2 & 0.3 & -6.8 & -8.9 \\
\hline Operating surplus/assets & -2.0 & -0.5 & 0.3 & 0.1 & -2.0 & -3.0 \\
\hline
\end{tabular}

Source: Ministry of Finance, Monitoring Cell, Finance Division.

1/ Does not include changes in inventories.

2/ Defined as after-tax retained income plus depreciation.

$3 /$ Includes arrears to government and changes in domestic bank debt (including arrears). 
Table 14. Bangladesh: Profits of Nonfinancial Public Corporations, 2000/01-2005/06 1/

(In millions of taka)

\begin{tabular}{|c|c|c|c|c|c|c|}
\hline & $2000 / 01$ & $2001 / 02$ & $2002 / 03$ & $2003 / 04$ & $2004 / 05$ & $2005 / 06$ \\
\hline \multicolumn{7}{|l|}{ Manufacturing } \\
\hline BTMC & -550 & -538 & -494 & -360 & -306 & -384 \\
\hline BJMC & $-3,811$ & $-2,915$ & $-2,055$ & $-2,114$ & $-1,743$ & $-1,105$ \\
\hline BSEC & 3 & 129 & 156 & 143 & 160 & 112 \\
\hline BSFIC & -469 & -633 & -981 & -586 & -176 & 662 \\
\hline $\mathrm{BCIC}$ & $-1,551$ & $-1,140$ & -733 & $-1,353$ & $-1,736$ & $-2,925$ \\
\hline BFIDC & -150 & -149 & -67 & 24 & 113 & 138 \\
\hline Sub-Total & $-6,527$ & $-5,247$ & $-4,174$ & $-4,247$ & $-3,688$ & $-3,502$ \\
\hline \multicolumn{7}{|c|}{ Electricity, gas, water } \\
\hline PDB & $-4,631$ & $-4,338$ & 1,746 & $-1,214$ & $-6,183$ & $-8,971$ \\
\hline DESA & $-3,694$ & $-4,587$ & $-2,599$ & $-1,672$ & -337 & -427 \\
\hline CWASA & -56 & -32 & 1 & 21 & 12 & 20 \\
\hline DWASA & 18 & 170 & 81 & 131 & 76 & 435 \\
\hline BOGMC & 1,968 & 1,993 & 3,492 & 3,772 & 0 & 0 \\
\hline Sub-Total & $-6,396$ & $-6,793$ & 2,721 & 1,039 & $-6,432$ & $-8,943$ \\
\hline \multicolumn{7}{|c|}{ Transport and communication } \\
\hline BSC & -125 & 1 & 2 & 153 & 333 & 403 \\
\hline BIWTC & 29 & 105 & 186 & 254 & 265 & 164 \\
\hline CPA & 1,310 & 1,353 & 1,019 & 1,618 & 2,351 & 1,763 \\
\hline CDWMB & 64 & 37 & 41 & 182 & -99 & -174 \\
\hline MPA & 138 & 123 & -109 & -126 & -155 & -165 \\
\hline MDWMB & -5 & -22 & -25 & -142 & -6 & -124 \\
\hline BLPA & $\ldots$ & 12 & 29 & 39 & 95 & 243 \\
\hline BIMAN & -997 & -741 & -611 & 173 & $-2,495$ & $-7,048$ \\
\hline BRTC & -158 & -237 & -329 & -419 & -353 & -257 \\
\hline JMBA & $\ldots$ & 708 & 720 & 1,062 & 1,433 & 1,634 \\
\hline Sub-Total & 256 & 1,339 & 922 & 2,793 & 1,368 & $-3,560$ \\
\hline \multicolumn{7}{|l|}{ Commercial } \\
\hline $\mathrm{BPC}$ & $-14,998$ & $-6,050$ & $-1,255$ & $-9,672$ & $-28,601$ & $-36,816$ \\
\hline BJC & 4 & 2 & 14 & 1 & 16 & 2 \\
\hline TCB & 79 & 668 & -94 & 18 & 19 & -832 \\
\hline Sub-Total & $-14,915$ & $-5,380$ & $-1,335$ & $-9,653$ & $-28,566$ & $-37,646$ \\
\hline \multicolumn{7}{|c|}{ Agriculture and fishery } \\
\hline BFDC(Fish) & -6 & 9 & -7 & 3 & 6 & -9 \\
\hline BADC & -255 & -283 & -265 & -213 & -112 & -132 \\
\hline Sub-Total & -261 & -274 & -271 & -210 & -106 & -141 \\
\hline \multicolumn{7}{|l|}{ Construction } \\
\hline CDA & 65 & 25 & 19 & 87 & 70 & 724 \\
\hline RAJUK & 156 & 112 & -27 & -24 & 489 & 275 \\
\hline KDA & 44 & 17 & 46 & 37 & 18 & -2 \\
\hline RDA & 12 & 12 & 19 & 32 & 32 & 0 \\
\hline Sub-Total & 275 & 167 & 57 & 133 & 608 & 997 \\
\hline \multicolumn{7}{|l|}{ Services } \\
\hline BFDC(Film) & 2 & 1 & -2 & 1 & 2 & 3 \\
\hline BFFWT & 218 & 37 & 44 & -17 & -83 & -65 \\
\hline BTB & 8 & 6 & 5 & -4 & 13 & 4 \\
\hline BIWTA & -523 & -483 & -178 & -70 & -190 & -173 \\
\hline BPRC & 112 & 6 & 4 & 6 & 6 & 6 \\
\hline BWDB & -20 & 22 & -1 & 34 & 267 & 259 \\
\hline REB & 296 & 1,347 & 930 & 1,756 & 2,586 & 2,245 \\
\hline BSCIC & -62 & -89 & -61 & -33 & -22 & -88 \\
\hline BEPZA & 179 & 202 & 265 & 476 & 523 & 333 \\
\hline CAAB & 1,041 & 889 & 911 & 788 & 773 & 370 \\
\hline $\mathrm{BHB}$ & 0 & 0 & 0 & 0 & 0 & 0 \\
\hline BSB & -15 & -19 & -18 & 0 & 2 & -4 \\
\hline EPB & 4 & 28 & 54 & 83 & 50 & -5 \\
\hline BTRC & $\ldots$ & 19 & 1,151 & 1,460 & 3,643 & 4,395 \\
\hline Sub-Total & 1,238 & 1,967 & 3,106 & 4,481 & 7,569 & 7,280 \\
\hline Total & $-26,329$ & $-14,222$ & 1,025 & $-5,663$ & $-29,246$ & $-45,516$ \\
\hline
\end{tabular}

Source: Ministry of Finance, Monitoring Cell, Finance Division.

$1 /$ Net profits after tax. 
Table 15. Bangladesh: Government Domestic Securities, 1999/00-2006/07

(In millions of taka; amount outstanding at end of period)

\begin{tabular}{|c|c|c|c|c|c|c|c|c|}
\hline & 1999/00 & $2000 / 01$ & $2001 / 02$ & $2002 / 03$ & $2003 / 04$ & $2004 / 05$ & $2005 / 06$ & Feb. 2007 \\
\hline Total securities outstanding & 361,448 & 432,871 & 508,261 & 502,993 & 532,576 & 547,933 & 572,297 & 661,071 \\
\hline National Savings Directorate instruments & 169,320 & 211,711 & 258,717 & 258,872 & 362,216 & 366,200 & 395,893 & 421,497 \\
\hline Savings certificates & 134,025 & 170,195 & 207,143 & 193,978 & 205,907 & 253,926 & 267,718 & 281,200 \\
\hline 8-year Defense Savings Certificate $1 /$ & 68,157 & 87,204 & 103,185 & 6,087 & 4,586 & 74,566 & 61,911 & 51,780 \\
\hline Family Savings Certificate $1 /$ & 9,581 & 12,456 & 15,017 & 94,245 & 84,883 & 6,187 & 3,155 & 1,021 \\
\hline Savings Certicate with 3 months profit & 25,851 & 40,394 & 59,659 & 19,623 & 26,949 & 126,719 & 133,239 & 142,778 \\
\hline 5-year Savings Certificate & 17,771 & 15,983 & 13,921 & 51 & 44 & 34,409 & 48,267 & 57,729 \\
\hline Jamanat Sanchaya Patra & 6 & 25 & 49 & 157 & 157 & 37 & 36 & 35 \\
\hline Pensioner's Sanchaypatra & $\ldots$ & $\ldots$ & $\ldots$ & $\ldots$ & $\ldots$ & 6,144 & 16,978 & 25,170 \\
\hline Post Office savings & 19,582 & 22,966 & 29,825 & 40,895 & 56,451 & 72,609 & 83,542 & 93,191 \\
\hline General & 1,157 & 2,043 & 2,696 & 3,412 & 4,202 & 5,055 & 5,198 & 5,716 \\
\hline Term & 17,637 & 19,911 & 25,922 & 36,057 & 50,574 & 65,528 & 75,939 & 84,741 \\
\hline Life insurance & 787 & 1,013 & 1,207 & 1,426 & 1,675 & 2,026 & 2,405 & 2,734 \\
\hline Wage Earner Development Bond & 9,204 & 10,559 & 11,926 & 12,298 & 15,390 & 22,012 & 25,863 & 28,608 \\
\hline 3-year National Investment Bond & 4,891 & 6,265 & 8,017 & 7,584 & 9,202 & 10,730 & 10,656 & 9,375 \\
\hline U.S. Dollar Premium Bond & $\ldots$ & $\ldots$ & $\ldots$ & 461 & 70,276 & 966 & 1,078 & 1,232 \\
\hline U.S. Dollar Investment Bond & $\ldots$ & $\ldots$ & $\ldots$ & 1,701 & 2,919 & 3,737 & 4,602 & 5,332 \\
\hline Treasury bills & 152,176 & 179,074 & 207,338 & 200,786 & 123,197 & 167,516 & 149,835 & 162,163 \\
\hline Bangladesh Bank holdings & 75,727 & 94,785 & 112,791 & 65,940 & 11,300 & 15,064 & 24,118 & 23,012 \\
\hline Treasury bills to increase reserve fund & 30 & 30 & 30 & 30 & 0 & 0 & 0 & 0 \\
\hline Treasury bills against demonetized Pakistani notes & 1,986 & 1,986 & 1,986 & 1,986 & 0 & 0 & 0 & 0 \\
\hline Government treasury bills & 73,711 & 92,769 & 110,775 & 63,924 & 11,300 & 15,064 & 24,118 & 23,012 \\
\hline Treasury bills auctioned to DMBs and nonbank public & 76,449 & 84,289 & 94,547 & 134,846 & 111,897 & 152,452 & 125,717 & 139,151 \\
\hline 28-day $2 /$ & 28,850 & 42,025 & 43,815 & 19,162 & 9,237 & 17,984 & 14,615 & 26,830 \\
\hline 91-day & 120 & 710 & 5,370 & 4,370 & 148 & 1,077 & 1,140 & 10,110 \\
\hline 182-day & 10,060 & 1,735 & 100 & 1,500 & 6,151 & 7,583 & 1,123 & 5,405 \\
\hline 364-day & 19,770 & 14,562 & 10,418 & 21,127 & 12,083 & 8,842 & 4,448 & 9,288 \\
\hline Two-year & 17,649 & 25,038 & 21,029 & 20,158 & 27,202 & 31,817 & 19,461 & 11,809 \\
\hline Five-year & 0 & 219 & 13,815 & 68,530 & 57,076 & 85,149 & 84,930 & 75,709 \\
\hline Original maturity 5 years and above & 38,195 & 40,586 & 40,166 & 41,515 & 43,620 & 5,365 & 12,832 & 40,960 \\
\hline
\end{tabular}

Sources: National Savings Directorate;and Bangladesh Bank.

1/ Discontinued July 2002.

2/ For maturity 28-day to 5 Year, up to March 2007. 
Table 16. Bangladesh: Central Bank Balance Sheet, 2001/02-2006/07

2001/02 2002/03 2003/04 2004/05 2005/06

\begin{tabular}{ccc}
\multicolumn{3}{c}{$2006 / 07$} \\
\hline Sep. $\quad$ Dec. Mar.
\end{tabular}

\begin{tabular}{|c|c|c|c|c|c|c|c|c|}
\hline \multirow[b]{2}{*}{ Net international reserves } & \multicolumn{8}{|c|}{ (End of period; in billions of taka) } \\
\hline & 46 & 94 & 116 & 130 & 155 & 152 & 171 & 199 \\
\hline Net domestic assets & 160 & 121 & 123 & 144 & 185 & 206 & 245 & 193 \\
\hline Net credit to central government & 108 & 62 & 110 & 145 & 237 & 222 & 258 & 225 \\
\hline Credit to other nonfinancial public sector & 1 & 1 & 1 & 0 & 0 & 0 & 0 & 0 \\
\hline Credit to deposit money banks & 47 & 48 & 55 & 57 & 60 & 60 & 60 & 60 \\
\hline Other items, net & 3 & 9 & -43 & -59 & -111 & -76 & -72 & -92 \\
\hline Reserve money & 206 & 214 & 239 & 274 & 340 & 358 & 417 & 392 \\
\hline Currency & 139 & 153 & 173 & 203 & 249 & 259 & 318 & 285 \\
\hline \multirow[t]{2}{*}{ Reserves } & 67 & 61 & 66 & 70 & 90 & 98 & 98 & 107 \\
\hline & \multicolumn{8}{|c|}{ (Annual change) } \\
\hline Net international reserves & 20 & 48 & 22 & 15 & 24 & 30 & 39 & 57 \\
\hline Net domestic assets & 23 & -39 & 2 & 21 & 41 & 48 & 66 & 21 \\
\hline Net credit to central government & 16 & -46 & 13 & 35 & 92 & 81 & 90 & 41 \\
\hline Credit to other nonfinancial public sector & 0 & 0 & 0 & -1 & 0 & 0 & 0 & 0 \\
\hline Credit to deposit money banks & 4 & 1 & 6 & 3 & 2 & 2 & 1 & 1 \\
\hline Other items, net & 4 & 6 & -16 & -16 & -52 & -36 & -25 & -20 \\
\hline Reserve money & 44 & 9 & 24 & 35 & 66 & 78 & 105 & 78 \\
\hline Currency & 10 & 15 & 19 & 30 & 46 & 51 & 95 & 49 \\
\hline Reserves & 33 & -6 & 5 & 5 & 20 & 27 & 10 & 29 \\
\hline \multicolumn{9}{|l|}{ Memorandum items: } \\
\hline Net international reserves (in millions of U.S. dollars) & 792 & 1,604 & 1,915 & 2,046 & 2,221 & 2,253 & 2,478 & 2,894 \\
\hline Required domestic cash reserves (in millions of taka) & 37 & 41 & 49 & 66 & 86 & 88 & 93 & 114 \\
\hline Excess domestic cash reserves (in millions of taka) & 30 & 20 & 17 & 4 & 4 & 10 & 5 & -7 \\
\hline
\end{tabular}

Sources: Data provided by the Bangladesh authorities; and IMF staff estimates. 
Table 17. Bangladesh: Monetary Survey, June 2002-March 2007 1/

\begin{tabular}{|c|c|c|c|c|c|c|c|c|}
\hline & \multirow{2}{*}{$2001 / 02$} & \multirow{2}{*}{$2002 / 03$} & \multirow{2}{*}{$2003 / 04$} & \multirow{2}{*}{$2004 / 05$} & \multirow{2}{*}{$2005 / 06$} & \multicolumn{3}{|c|}{$2006 / 07$} \\
\hline & & & & & & Sep. & Dec. & Mar. \\
\hline & \multicolumn{8}{|c|}{ (End of period; in billions of taka) } \\
\hline Net foreign assets & 96 & 140 & 163 & 186 & 218 & 223 & 251 & 277 \\
\hline Bangladesh Bank & 46 & 94 & 116 & 130 & 155 & 152 & 171 & 199 \\
\hline Commercial banks & 50 & 47 & 47 & 55 & 63 & 71 & 80 & 78 \\
\hline Net domestic assets & 891 & 1,000 & 1,134 & 1,328 & 1,588 & 1,640 & 1,760 & 1,733 \\
\hline Domestic credit & 957 & 1,034 & 1,219 & 1,431 & 1,732 & 1,797 & 1,901 & 1,863 \\
\hline Net credit to central government & 184 & 173 & 204 & 237 & 296 & 320 & 347 & 326 \\
\hline Credit to other nonfinancial public sector & 59 & 55 & 69 & 86 & 127 & 130 & 131 & 126 \\
\hline Credit to private sector & 715 & 806 & 946 & 1107 & 1309 & 1347 & 1,423 & 1,453 \\
\hline Other items, net $1 /$ & -67 & -34 & -85 & -102 & -144 & -157 & -141 & -130 \\
\hline Broad money (M2) & 986 & 1,140 & 1,297 & 1,514 & 1,806 & 1,863 & 2,010 & 2,010 \\
\hline Currency in circulation & 125 & 139 & 158 & 185 & 229 & 241 & 300 & 273 \\
\hline \multirow[t]{2}{*}{ Deposits } & 861 & 1,001 & 1,139 & 1,329 & 1,578 & 1,622 & 1,711 & 1,737 \\
\hline & \multicolumn{8}{|c|}{ (Year-on-year percent change) } \\
\hline Net foreign assets & 25.8 & 46.8 & 16.3 & 14.2 & 17.2 & 23.8 & 35.4 & 41.2 \\
\hline Net domestic assets & 11.9 & 12.2 & 13.4 & 17.1 & 19.6 & 19.2 & 20.5 & 16.8 \\
\hline Domestic credit & 13.2 & 8.0 & 11.9 & 17.4 & 21.1 & 20.2 & 21.0 & 15.1 \\
\hline Net credit to central government & 13.4 & -5.8 & 7.8 & 16.4 & 24.8 & 29.9 & 37.0 & 32.2 \\
\hline Credit to other nonfinancial public sector & 4.8 & -5.7 & 23.8 & 25.8 & 46.7 & 25.1 & 4.2 & -0.4 \\
\hline Credit to private sector & 13.9 & 12.6 & 12.0 & 17.0 & 18.3 & 17.7 & 19.4 & 16.6 \\
\hline Other items, net & 34.2 & -48.8 & -32.3 & 20.9 & 40.2 & 32.2 & 28.2 & -4.2 \\
\hline \multirow[t]{2}{*}{ Broad money (M2) } & 13.1 & 15.6 & 13.8 & 16.7 & 19.3 & 19.7 & 22.2 & 19.7 \\
\hline & \multicolumn{8}{|c|}{ (In billions of taka, unless otherwise noted) } \\
\hline \multicolumn{9}{|l|}{ Memorandum items: } \\
\hline Broad money multiplier & 4.79 & 5.32 & 5.43 & 5.53 & 5.32 & 5.20 & 4.83 & 5.16 \\
\hline Broad money velocity & 2.77 & 2.64 & 2.58 & 2.45 & 2.31 & 2.54 & 2.36 & 2.36 \\
\hline Net domestic financing of central government $2 /$ & 69 & 38 & 60 & 63 & 86 & 34 & 68 & 42 \\
\hline Bank & 22 & -11 & 13 & 34 & 59 & 24 & 51 & 30 \\
\hline Nonbanks & 47 & 48 & 46 & 29 & 28 & 10 & 17 & 12 \\
\hline
\end{tabular}

Sources: Data provided by the Bangladesh authorities; and IMF staff estimates.

1/ In April 2004, Bangladesh Bank revised its accounting standards in line with IAS best practices. Accordingly, percent changes are adjusted to maintain consistency.

2/ Since beginning of fiscal year. 
Table 18. Bangladesh: Reserves of Deposit Money Banks, 1999/00-2005/06 1/

\begin{tabular}{|c|c|c|c|c|c|c|c|}
\hline & $1999 / 00$ & $2000 / 01$ & $2000 / 02$ & $2002 / 03$ & $2003 / 04$ & $2004 / 05$ & $2005 / 06$ \\
\hline & \multicolumn{7}{|c|}{ (In billions of taka) } \\
\hline Required liquid assets $2 /$ & 123 & 148 & 159 & 183 & 165 & 219 & 287 \\
\hline Nationalized commercial banks & 77 & 88 & 92 & 100 & 81 & 97 & 115 \\
\hline Private banks & 35 & 48 & 54 & 68 & 70 & 104 & 146 \\
\hline Foreign banks & 11 & 12 & 13 & 15 & 14 & 18 & 26 \\
\hline Actual liquid assets & 174 & 182 & 220 & 257 & 278 & 306 & 367 \\
\hline Nationalized commercial banks & 98 & 102 & 124 & 124 & 128 & 117 & 126 \\
\hline Private commercial banks & 52 & 57 & 73 & 103 & 119 & 143 & 193 \\
\hline Foreign commercial banks & 24 & 23 & 23 & 30 & 31 & 46 & 48 \\
\hline Excess liquidity & 50 & 40 & 62 & 75 & 113 & 87 & 80 \\
\hline Nationalized commercial banks & 21 & 17 & 32 & 25 & 46 & 20 & 11 \\
\hline Private commercial banks & 16 & 12 & 19 & 35 & 50 & 39 & 47 \\
\hline Foreign commercial banks & 13 & 11 & 11 & 15 & 17 & 28 & 22 \\
\hline Total deposits & 633 & 734 & 827 & 957 & 1,075 & 1,465 & 1,760 \\
\hline Nationalized commercial banks & 382 & 422 & 459 & 498 & 509 & 621 & 654 \\
\hline Private commercial banks & 195 & 250 & 303 & 384 & 480 & 731 & 955 \\
\hline Foreign commercial banks & 56 & 62 & 65 & 75 & 86 & 113 & 150 \\
\hline \multicolumn{8}{|l|}{ Memorandum items: } \\
\hline Excess liquidity & 7.9 & 5.4 & 7.5 & 7.8 & 10.5 & 5.9 & 4.5 \\
\hline Nationalized commercial banks & 5.5 & 4.0 & 7.0 & 5.0 & 9.0 & 3.2 & 1.7 \\
\hline Private commercial banks & 8.2 & 4.8 & 6.3 & 9.1 & 10.4 & 5.3 & 4.9 \\
\hline Foreign commercial banks & 23.2 & 17.7 & 16.9 & 30.0 & 19.8 & 24.8 & 14.7 \\
\hline
\end{tabular}

Source: Bangladesh Bank, Department of Banking Operations and Development.

1/ Data refer to the last Thursday of the month.

2/ Liquid asset requirements against all deposits, including government and interbank deposits, were 25 percent from October 29, 1987; 24 percent from April 4, 1991; 23 percent from April 25, 1991; 20 percent from May 28, 1992; and 16 percent from November 8, 2003 to the present. 
Table 19. Bangladesh: Interest Rate Structure of the Banking Sector, 2002/03-2006/07

(In percent per annum; end of period)

\begin{tabular}{|c|c|c|c|c|c|}
\hline & $2002 / 031 /$ & $2003 / 04$ & $2004 / 05$ & $2005 / 06$ & $2006 / 07$ 2/ \\
\hline Bangladesh Bank discount rate & 6.0 & 5.0 & 5.0 & 5.0 & 5.0 \\
\hline \multicolumn{6}{|l|}{ Scheduled banks' deposit rates } \\
\hline Deposit rate: 3-6 mos. average & 7.4 & 6.4 & 8.1 & 9.4 & 9.2 \\
\hline Savings rate & 6.7 & 4.8 & 5.5 & 6.0 & 6.0 \\
\hline \multicolumn{6}{|l|}{ Scheduled banks' lending rates } \\
\hline Agricultural maximum rate & 16.0 & 15.0 & 14.0 & 16.0 & 16.0 \\
\hline Commercial maximum rate & 24.0 & 24.0 & 14.0 & 17.0 & 17.0 \\
\hline Small loan scheme & 18.0 & 24.0 & 22.5 & 22.5 & 22.5 \\
\hline Call money lending rate & 5.2 & 5.4 & 6.8 & 11.1 & 7.2 \\
\hline \multicolumn{6}{|l|}{ Selected treasury bill rates } \\
\hline 28-day treasury bill yield & 6.4 & 4.0 & 6.6 & 7.1 & 7.3 \\
\hline 91-day treasury bill yield & 7.4 & 5.0 & 6.7 & 7.4 & 7.6 \\
\hline
\end{tabular}

Sources: Bangladesh Bank; and IMF Statistics Department.

1/ August 2003.

2/ December 2006. 
Table 20. Bangladesh: Interest Rate Structure of Nonbank Financial Institutions and Government Debt, 1999/00-2006/07

( In percent)

1999/00 2000/01 2001/02 2002/03 2003/04 2004/05 2005/06 $\quad$ March 2007

Interest rates on deposits with Post Office Savings Bank

Ordinary account

Fixed deposit account

1 -year

2- years

3- years

Interest rates on government ways and means advances

Interest rates on selected savings instruments

8-year Defense Savings Certificates

Family Savings Certificate

$\begin{array}{rrrrrrrr}8.5 & 8.5 & 8.5 & 8.5 & 8.5 & 7.5 & 7.5 & 7.5 \\ & & & & & & & \\ 10.5 & 10.5 & 10.5 & 10.5 & 10.5 & 9.5 & 10.0 & 10.0 \\ 11.5 & 11.5 & 11.5 & 11.5 & 11.5 & 10.5 & 11.0 & 11.0 \\ 12.5 & 12.5 & 12.5 & 12.5 & 12.5 & 11.5 & 12.0 & 12.0 \\ 6.5 & 6.5 & 6.5 & 5.5 & 4.5 & 4.5 & 4.5 & 6.5\end{array}$

6-month Profit Savings Certificate

3-month Profit Savings Certificate

$\begin{array}{lll}17.8 & 17.8 & 15.8\end{array}$

$\begin{array}{lll}13.2 & 13.2 & 11.9\end{array}$

5-year Savings Certificates

$13.5 \quad 13.5 \quad 12.0$

$13.5 \quad 13.5 \quad 12.0$

5-year Wage Earner's Development Bonds

3-year National Investment Bond

14.5

13.5

12.5

15.8

15.8

15.8

12.5

8.5

8.5

8.5

U.S. Dollar Premium Bond

U.S. Dollar Investment Bond

$\begin{array}{rrrr}\ldots & \ldots & \ldots & 7.5\end{array}$

7.5

6.5

$\begin{array}{rrrr}\ldots & \ldots & \ldots & \ldots \\ \ldots & \ldots & \ldots & \ldots \\ \ldots & \ldots & \ldots & \ldots \\ 10.5 & 10.0 & 11.5 & 11.5 \\ 11.0 & 10.5 & 12.0 & 12.0 \\ 15.8 & 15.8 & 15.8 & 15.8 \\ 8.5 & 8.5 & 8.5 & 8.5 \\ 7.5 & 7.5 & 7.5 & 7.8 \\ 6.5 & 6.5 & 6.5 & 6.5 \\ & & & \end{array}$

Source: Bangladesh Bank. 
Table 21. Bangladesh: Financial System, December 2006

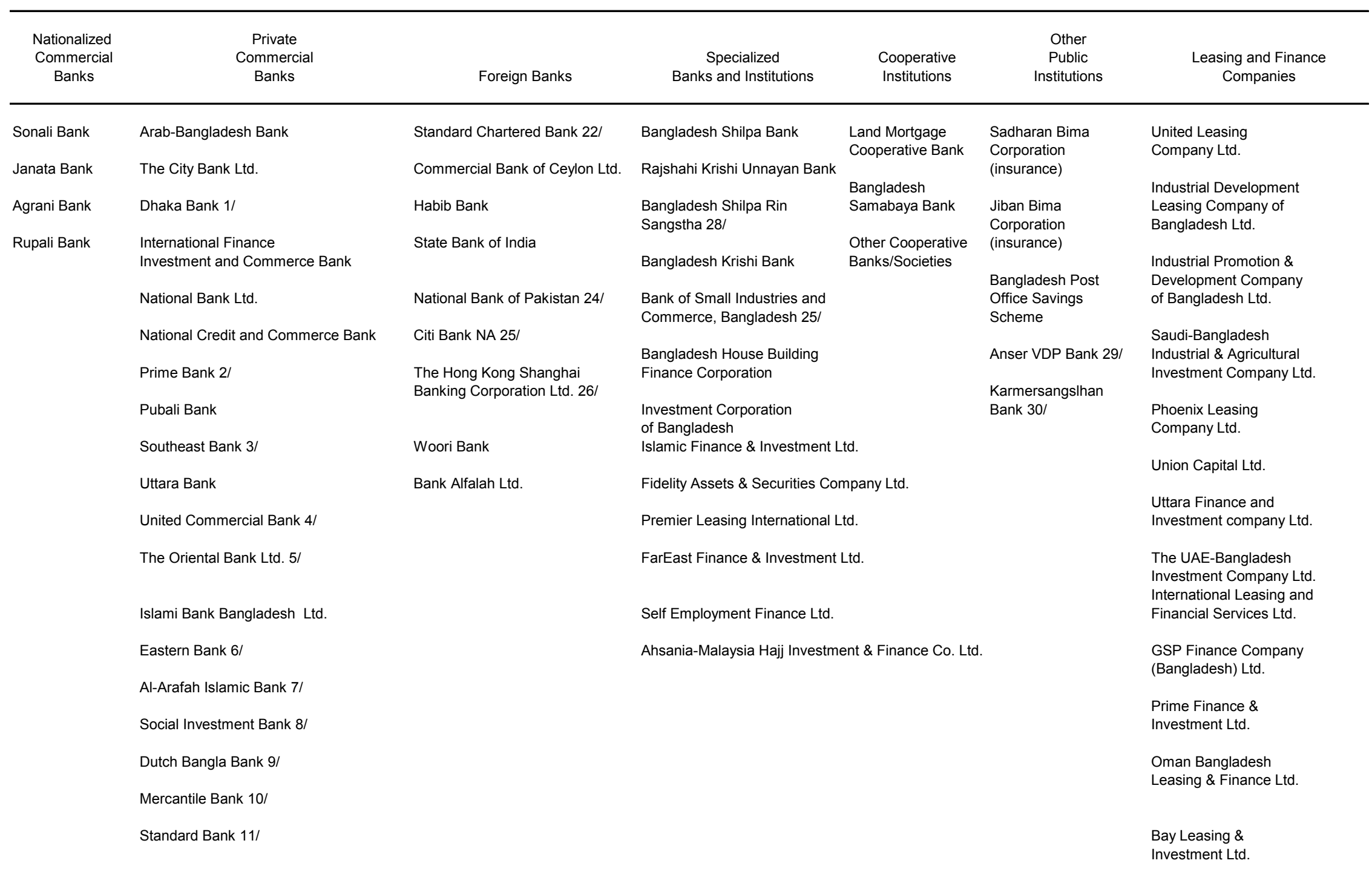


Table 21. Bangladesh: Financial System, December 2006 (concluded)

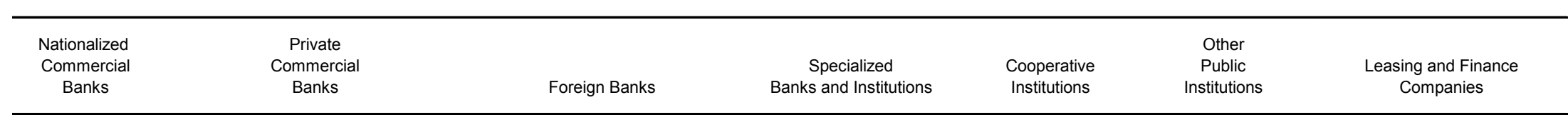

Export Import Bank of Bangladesh 12/

One Bank 13/

Delta-BRAC Housing

Bangladesh Commerce Bank 14

Finance Corporation Ltd.

The Mutual Trust Bank 15/

Lanka Bangla Finance Ltd.

First Security Bank 16/

Peoples Leasing \&

Financial Services Ltd.

The Premier Bank 17/

Infrastructure Development

Company Ltd. (IDCOL)

Bank Asia 18/

Bangladesh Industrial

Finance Company Ltd.

The Trust Bank 11/

National Housing

Shahjalal Bank Ltd. 19/

Finance and Inv. Ltd.

Jamuna Bank Ltd. 20/

Midas Financing Ltd.

BRAC Bank Ltd. 21/

First Lease International Ltd.

Bangladesh Finance and

Investment Ltd.

Industrial and Infrastructure

Development Finance

Co. Ltd.

Sources: Bangladesh Bank, Department of Banking Operation; and Development and Research Department.

1/ Effective July 5, 1995.

2/ Effective April 17, 1995.

3/ Effective May 25, 1995

4/Effective September 21, 1994

5/ Former Al-Baraka Bank Ltd. Islamic banking practices.

6/ Former Bank of Credit and Commerce Int. Ltd. was

converted into Eastern Bank Ltd. effective August 16, 1992.

7/ Effective September 25, 1995.

8/ Effective November 22, 1995.

9/ Effective June 3, 1996.

10/ Effective June 2, 1999

11/ Opened on June 3, 1999
12/ Effective August 3, 1999.

13/ Effective July 14, 1999.

14/ Effective September 16, 1999

15/ Effective October 24, 1999.

16/ Effective October 25, 1999.

17/ Effective October 26, 1999.

18/ Effective November 27, 1999. The Bank Nova Scotia

was merged with Bank Asia from February 15, 2001.

19/ Effective May 10, 2001

20/ Effective June 3, 2001.

21/ Effective July 2, 2001.

22/ Former ANZ Grindlays Bank merged with Standard

Chartered Bank on September 24, 2000.
24/ Effective August 31, 1994

25/ Effective June 24, 1995

26/ Effective December 3, 1996.

28/ Bangladesh Shilpa Rin Sangstha was converted into a specialized bank

effective February 9, 1993.

29/ Effective November 1996.

30/ Effective September 22, 1998

\section{CInternational Monetary Fund. Not for Redistribution}


Table 22. Bangladesh: Financial System Structure, 1999-2006

\begin{tabular}{|c|c|c|c|c|c|c|c|c|}
\hline & \multicolumn{8}{|c|}{ December } \\
\hline & 1999 & 2000 & 2001 & 2002 & 2003 & 2004 & 2005 & 2006 \\
\hline \multicolumn{9}{|c|}{ Structure of the financial system (number) } \\
\hline Banks & 39 & 49 & 51 & 50 & 50 & 49 & 48 & 48 \\
\hline Nationalized commercial banks & 4 & 4 & 4 & 4 & 4 & 4 & 4 & 4 \\
\hline Specialized development banks & 5 & 5 & 5 & 5 & 5 & 5 & 5 & 5 \\
\hline Private commercial banks & 17 & 30 & 30 & 30 & 30 & 30 & 30 & 30 \\
\hline Foreign commercial banks & 13 & 10 & 12 & 11 & 11 & 10 & 9 & 9 \\
\hline Insurance companies & 32 & $\ldots$ & 62 & $\ldots$ & $\ldots$ & $\ldots$ & 62 & $\ldots$ \\
\hline Life & 7 & $\ldots$ & 18 & $\ldots$ & $\ldots$ & $\ldots$ & 44 & $\ldots$ \\
\hline Non-life & 25 & $\ldots$ & 44 & $\ldots$ & $\ldots$ & $\ldots$ & 18 & $\ldots$ \\
\hline Finance companies & 19 & $\ldots$ & 26 & $\ldots$ & $\ldots$ & $\ldots$ & 28 & 23 \\
\hline Microfinance institutions & $\ldots$ & $\ldots$ & 624 & $\ldots$ & $\ldots$ & $\ldots$ & $1,000+$ & $\ldots$ \\
\hline \multicolumn{9}{|l|}{ Financial system assets (in billion taka) } \\
\hline Banks & $\ldots$ & $\ldots$ & 1,444 & 1,538 & 1,636 & 1,913 & 2,254 & 2,589 \\
\hline Nationalized commercial banks & $\ldots$ & $\ldots$ & 635 & 716 & 701 & 771 & 849 & 875 \\
\hline Specialized development banks & $\ldots$ & $\ldots$ & 141 & 114 & 100 & 127 & 141 & 156 \\
\hline Private commercial banks & $\ldots$ & $\ldots$ & 495 & 557 & 658 & 844 & 1,033 & 1,285 \\
\hline Foreign commercial banks & $\ldots$ & $\ldots$ & 174 & 152 & 177 & 172 & 231 & 272 \\
\hline Insurance companies & $\ldots$ & $\ldots$ & 36 & $\ldots$ & $\ldots$ & $\ldots$ & $\ldots$ & $\ldots$ \\
\hline Life & $\ldots$ & $\ldots$ & 23 & $\ldots$ & $\ldots$ & $\ldots$ & $\ldots$ & $\ldots$ \\
\hline Non-Life & $\ldots$ & $\ldots$ & 13 & $\ldots$ & $\ldots$ & $\ldots$ & $\ldots$ & $\ldots$ \\
\hline Nonbank financial institutions & $\ldots$ & $\ldots$ & 19 & $\ldots$ & $\ldots$ & $\ldots$ & $\ldots$ & $\ldots$ \\
\hline Microfinance institutions & $\ldots$ & $\ldots$ & 38 & 36 & 43 & 53 & 65 & $\ldots$ \\
\hline (as percent of private sector credit) & $\ldots$ & $\ldots$ &  & 5 & 5 & 5 & 5 & - \\
\hline Securities market capitalization (DSE) & 44 & 61 & 64 & 69 & 98 & 224 & 215 & 266 \\
\hline
\end{tabular}

Source: Bangladesh Bank, Banking Regulation and Policy Department. 
Table 23. Bangladesh: Summary Performance Indicators in the Banking System, 1999-2006

(In percent; end of period)

\begin{tabular}{|c|c|c|c|c|c|c|c|}
\hline & 1999 & 2000 & 2002 & 2003 & 2004 & 2005 & 2006 \\
\hline Classified loans by group of bank $1 /$ & 36 & 30 & 28 & 22 & 18 & 14 & 13 \\
\hline Domestic banks & 39 & 32 & 30 & 24 & 18 & 12 & 12 \\
\hline Nationalized commercial banks & 46 & 39 & 34 & 29 & 25 & 21 & 23 \\
\hline Specialized development banks & 65 & 63 & 56 & 47 & 43 & 35 & 34 \\
\hline Private domestic banks & 27 & 22 & 17 & 12 & 9 & 6 & 5 \\
\hline Foreign banks & 4 & 3 & 3 & 3 & 2 & 1 & 1 \\
\hline Total classified loans by category $1 /$ & 36 & 30 & 28 & 22 & 18 & 11 & 13 \\
\hline Substandard 2/ & 5 & 4 & 9 & 10 & 7 & 9 & 13 \\
\hline Doubtful 2/ & 9 & 7 & 5 & 9 & 7 & 7 & 7 \\
\hline Bad $2 /$ & 86 & 89 & 86 & 81 & 86 & 84 & 80 \\
\hline \multicolumn{8}{|l|}{ Loan market shares $1 /$} \\
\hline Domestic banks & 93 & 93 & 94 & 93 & 93 & 94 & 93 \\
\hline Nationalized commercial and development banks & 58 & 55 & 55 & 51 & 47 & 45 & 41 \\
\hline Private domestic banks & 35 & 38 & 39 & 42 & 46 & 49 & 52 \\
\hline Foreign banks & 7 & 7 & 6 & 7 & 7 & 6 & 7 \\
\hline \multicolumn{8}{|l|}{ Actual provisioning in percent of required provisioning } \\
\hline Domestic banks & 43 & 46 & 56 & $\ldots$ & 30 & 42 & 46 \\
\hline Nationalized commercial banks & 36 & 37 & 31 & 7 & 7 & 25 & 30 \\
\hline Private domestic banks & 61 & 68 & 82 & 75 & 83 & 87 & 82 \\
\hline Foreign banks & 121 & 123 & 127 & 126 & 124 & 144 & 137 \\
\hline
\end{tabular}

Source: Bangladesh Bank, Banking Regulation and Policy Department.

$1 /$ In percent of gross loans.

2/ In percent of classified loans. 
Table 24. Bangladesh: Banks' Liquidity Positions, 2001-06

(In millions of taka; end of period)

\begin{tabular}{|c|c|c|c|c|c|c|}
\hline & 2001 & 2002 & 2003 & 2004 & 2005 & 2006 \\
\hline \multicolumn{7}{|l|}{ Nationalized commercial banks } \\
\hline Total deposits (incl. govt. deposits) 1/ & 473,570 & 515,060 & 524,987 & 567,514 & 621,306 & 654,126 \\
\hline Demand deposits (excl. govt. and DMB deposits) & 63,691 & 76,606 & 79,879 & 88,231 & 101,506 & 111,432 \\
\hline Time deposits (excl. govt. and DMB deposits) & 290,812 & 404,772 & 412,832 & 439,234 & 466,146 & 486,204 \\
\hline Total liquid assets (cash and eligible securities) & 114,657 & 132,503 & 123,584 & 122,272 & 117,544 & 126,227 \\
\hline Deposits in Bangladesh Bank & 40,179 & 31,867 & 29,259 & 23,027 & 33,675 & 34,260 \\
\hline Cash in vault & 4,789 & 5,218 & 5,175 & 5,597 & 5,464 & 6,777 \\
\hline Eligible securities & 66,583 & 95,418 & 89,150 & 93,648 & 78,405 & 85,190 \\
\hline Statutory liquidity requirement & 89,194 & 97,205 & 81,005 & 85,733 & 105,800 & 113,326 \\
\hline Excess/(shortfall) liquid assets & 25,463 & 35,298 & 42,579 & 36,539 & 11,744 & 12,901 \\
\hline Borrowing from Bangladesh Bank & 644 & 479 & 140 & 135 & 111 & 107 \\
\hline \multicolumn{7}{|l|}{ Private commercial banks } \\
\hline Total deposits (incl. govt. deposits) $1 /$ & 303,258 & 377,078 & 468,141 & 587,985 & 731,324 & 955,480 \\
\hline Demand deposits (excl. govt. and DMB deposits) & 60,781 & 72,594 & 82,147 & 98,774 & 124,645 & 152,789 \\
\hline Time deposits (excl. govt. and DMB deposits) & 210,392 & 299,184 & 378,872 & 481,002 & 595,671 & 789,305 \\
\hline Total liquid assets (cash and eligible securities) & 67,332 & 35,017 & 110,493 & 126,954 & 143,636 & 192,787 \\
\hline Deposits in Bangladesh Bank & 27,655 & 35,017 & 42,808 & 45,367 & 58,606 & 76,393 \\
\hline Cash in vault & 6,566 & 6,763 & 6,952 & 8,038 & 9,895 & 11,793 \\
\hline Eligible securities & 29,634 & 50,309 & 60,732 & 73,549 & 75,134 & 104,600 \\
\hline Statutory liquidity requirement & 50,020 & 62,421 & 66,171 & 78,727 & 108,933 & 141,976 \\
\hline Excess/(shortfall) liquid assets & 17,312 & 29,668 & 44,321 & 48,227 & 34,703 & 50,810 \\
\hline Borrowing from Bangladesh Bank & 272 & 0 & 1,903 & 675 & 4,678 & 6,449 \\
\hline \multicolumn{7}{|l|}{ Foreign commercial banks } \\
\hline Total deposits (incl. govt. deposits) $1 /$ & 64,411 & 71,858 & 84,496 & 95,525 & 112,684 & 150,816 \\
\hline Demand deposits (excl. govt. and DMB deposits) & 27,269 & 29,536 & 34,514 & 38,886 & 49,608 & 60,991 \\
\hline Time deposits (excl. govt. and DMB deposits) & 36,364 & 40,239 & 49,659 & 56,357 & 63,067 & 89,366 \\
\hline Total liquid assets (cash and eligible securities) & 21,676 & 15,668 & 31,880 & 35,168 & 46,767 & 48,166 \\
\hline Deposits in Bangladesh Bank & 8,911 & 15,668 & 13,971 & 14,029 & 21,149 & 23,724 \\
\hline Cash in vault & 762 & 822 & 812 & 1,278 & 2,357 & 2,552 \\
\hline Eligible securities & 12,003 & 12,282 & 17,097 & 19,860 & 22,261 & 21,890 \\
\hline Statutory liquidity requirement & 12,589 & 13,721 & 13,415 & 14,786 & 19,734 & 25,210 \\
\hline Excess/(shortfall) liquid assets & 9,087 & 15,051 & 18,466 & 20,382 & 26,034 & 22,956 \\
\hline Borrowing from Bangladesh Bank & 81 & 28 & 4 & 0 & 0 & 0 \\
\hline \multicolumn{7}{|l|}{ Specialized development banks } \\
\hline Total deposits (incl. govt. deposits) $1 /$ & 49,174 & 59,529 & 62,643 & 75,103 & 89,450 & 100,205 \\
\hline Demand deposits (excl. govt. and DMB deposits) & 5,693 & 7,441 & 7,787 & 8,761 & 10,989 & 12,907 \\
\hline Time deposits (excl. govt. and DMB deposits) & 36,345 & 49,660 & 52,058 & 62,526 & 68,618 & 78,000 \\
\hline Total liquid assets (cash and eligible securities) & 7,275 & 7,653 & 7,416 & 8,304 & 11,737 & 4,543 \\
\hline Deposits in Bangladesh Bank & 2,818 & 3,677 & 3,131 & 3,943 & 4,711 & 1,294 \\
\hline Cash in vault & 364 & 463 & 482 & 548 & 576 & 229 \\
\hline Eligible securities & 3,827 & 3,513 & 3,803 & 3,813 & 6,450 & 3,020 \\
\hline Statutory liquidity requirement & 3,070 & 3,791 & 3,808 & 4,831 & 6,521 & 4,133 \\
\hline Excess/(shortfall) liquid assets & 4,205 & 3,861 & 3,607 & 3,473 & 5,217 & 410 \\
\hline Borrowing from Bangladesh Bank & 47,781 & 49,123 & 49,232 & 58,026 & 62,045 & 63,106 \\
\hline \multicolumn{7}{|l|}{ All banks } \\
\hline Total deposits (incl. govt. deposits) $1 /$ & 890,414 & $1,023,526$ & $1,140,267$ & $1,326,127$ & $1,554,765$ & $1,860,626$ \\
\hline Demand deposits (excl. govt. and DMB deposits) & 157,434 & 186,176 & 204,327 & 234,651 & 286,748 & 338,118 \\
\hline Time deposits (excl. govt. and DMB deposits) & 573,913 & 793,855 & 893,421 & $1,039,119$ & $1,193,502$ & $1,442,875$ \\
\hline Total liquid assets (cash and eligible securities) & 210,940 & 190,840 & 273,372 & 292,698 & 318,684 & 371,722 \\
\hline Deposits in Bangladesh Bank & 79,564 & 86,228 & 89,169 & 86,366 & 118,141 & 135,671 \\
\hline Cash in vault & 12,481 & 13,266 & 13,421 & 15,462 & 18,292 & 21,351 \\
\hline Eligible securities & 112,047 & 161,522 & 170,782 & 190,871 & 182,251 & 214,700 \\
\hline Statutory liquidity requirement & 154,872 & 177,138 & 164,399 & 184,077 & 240,987 & 284,644 \\
\hline Excess/(shortfall) liquid assets & 56,068 & 83,878 & 108,973 & 108,621 & 77,697 & 87,078 \\
\hline Borrowing from Bangladesh Bank & 48,778 & 49,630 & 51,278 & 58,836 & 66,834 & 69,662 \\
\hline
\end{tabular}

Source: Bangladesh Bank, Department of Banking Operation and Development.

1/ As defined for purposes of Statutory Liquidity Requirement. 
Table 25. Bangladesh: Banks' Foreign Exchange Positions, 1999-2006

(In millions of U.S. dollars; end-December)

\begin{tabular}{|c|c|c|c|}
\hline & $\begin{array}{c}\text { Foreign Exchange } \\
\text { Liabilities }\end{array}$ & $\begin{array}{c}\text { Foreign Exchange } \\
\text { Assets }\end{array}$ & Net Position \\
\hline \multicolumn{4}{|c|}{ Nationalized commercial banks } \\
\hline 1999 & 296 & 553 & 257 \\
\hline 2000 & 323 & 563 & 240 \\
\hline 2001 & 348 & 502 & 154 \\
\hline 2002 & 399 & 517 & 118 \\
\hline 2003 & 218 & 412 & 194 \\
\hline 2004 & 152 & 423 & 271 \\
\hline 2005 & 204 & 442 & 238 \\
\hline 2006 & 312 & 525 & 213 \\
\hline \multicolumn{4}{|c|}{ Specialized development banks } \\
\hline 1999 & 0 & 8 & 8 \\
\hline 2000 & 0 & 23 & 23 \\
\hline 2001 & 0 & 18 & 18 \\
\hline 2002 & 1 & 19 & 18 \\
\hline 2003 & 0 & 11 & 11 \\
\hline 2004 & 2 & 8 & 6 \\
\hline 2005 & 2 & 18 & 16 \\
\hline 2006 & 2 & 22 & 20 \\
\hline \multicolumn{4}{|c|}{ Private commercial banks } \\
\hline 1999 & 71 & 258 & 187 \\
\hline 2000 & 144 & 402 & 258 \\
\hline 2001 & 165 & 335 & 170 \\
\hline 2002 & 107 & 256 & 149 \\
\hline 2003 & 92 & 255 & 163 \\
\hline 2004 & 107 & 280 & 173 \\
\hline 2005 & 127 & 285 & 158 \\
\hline 2006 & 141 & 327 & 186 \\
\hline \multicolumn{4}{|c|}{ Foreign commercial banks } \\
\hline 1999 & 97 & 93 & -4 \\
\hline 2000 & 102 & 216 & 114 \\
\hline 2001 & 159 & 226 & 67 \\
\hline 2002 & 119 & 124 & 5 \\
\hline 2003 & 129 & 98 & -31 \\
\hline 2004 & 84 & 121 & 37 \\
\hline 2005 & 90 & 167 & 77 \\
\hline 2006 & 104 & 243 & 139 \\
\hline \multicolumn{4}{|c|}{ All banks } \\
\hline 1999 & 464 & 912 & 448 \\
\hline 2000 & 569 & 1,204 & 635 \\
\hline 2001 & 672 & 1,081 & 409 \\
\hline 2002 & 626 & 916 & 290 \\
\hline 2003 & 439 & 776 & 337 \\
\hline 2004 & 345 & 832 & 487 \\
\hline 2005 & 423 & 912 & 489 \\
\hline 2006 & 559 & 1,117 & 558 \\
\hline
\end{tabular}

Source: Bangladesh Bank, Statistics Department. 
Table 26. Bangladesh: Bank's Reported Capital, 2001-06

(In billions of taka)

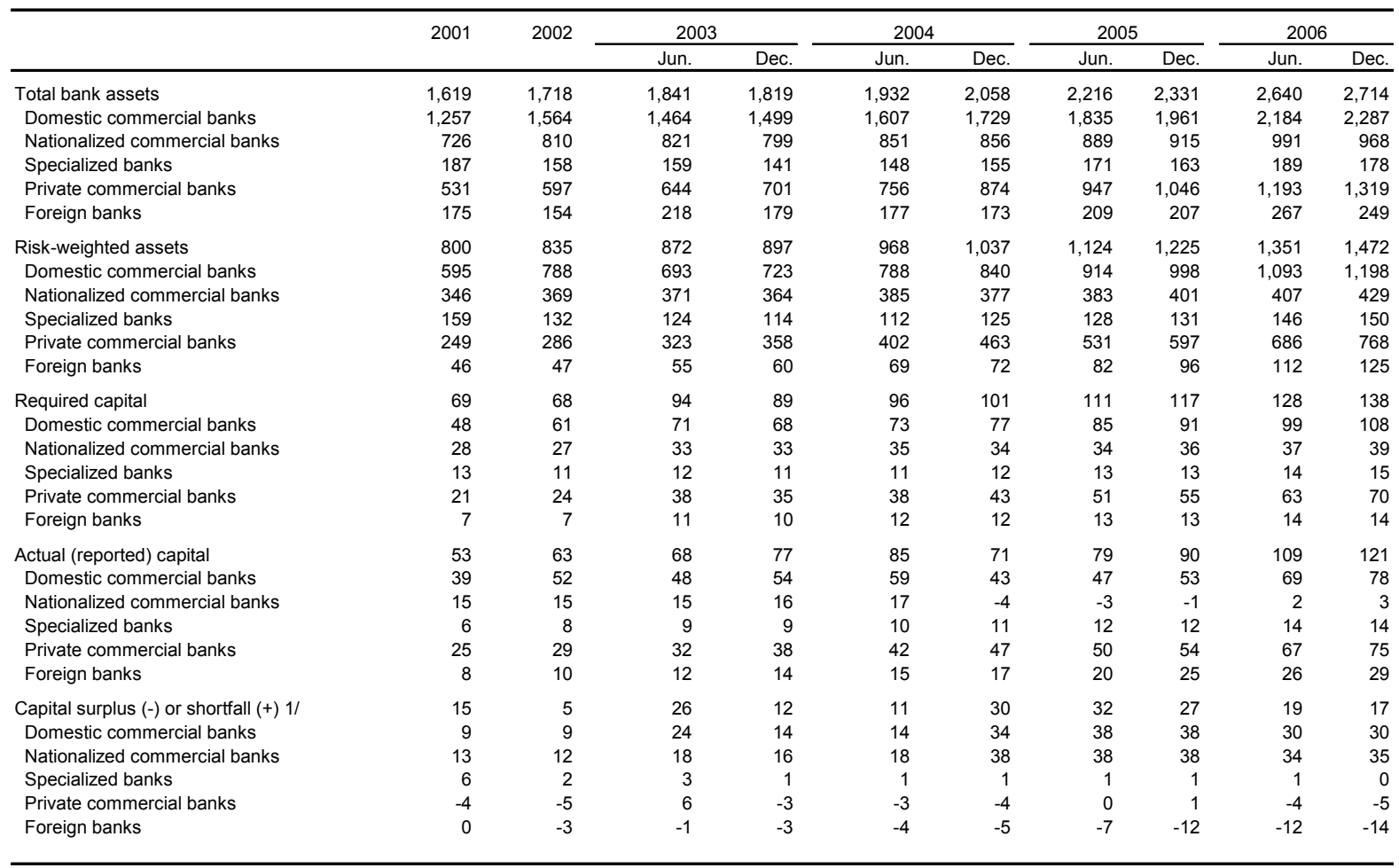

Sources: Bangladesh Bank, Bank Regulation and Policy Department (BRPD); and Off-site Supervision Department (OSD).

$1 /$ This capital shortfall calculation does not net out provision shortfall and accumulated losses. 
Table 27. Bangladesh: Balance of Payments, 2000/01-2005/06 1/

(In millions of U.S. dollars)

\begin{tabular}{|c|c|c|c|c|c|c|}
\hline & $2000 / 01$ & $2001 / 02$ & $2002 / 03$ & $2003 / 04$ & $2004 / 05$ & $2005 / 06$ \\
\hline Trade balance & $-2,011$ & $-1,768$ & $-2,215$ & $-2,319$ & $-3,297$ & $-2,879$ \\
\hline Exports (f.o.b.) & 6,419 & 5,929 & 6,492 & 7,521 & 8,573 & 10,422 \\
\hline Imports (f.o.b.) & $-8,430$ & $-7,697$ & $-8,707$ & $-9,840$ & $-11,870$ & $-13,301$ \\
\hline Services & -914 & -499 & -691 & -874 & -870 & $-1,110$ \\
\hline Income & -264 & -402 & -358 & -374 & -680 & -786 \\
\hline Transfers & 2,171 & 2,826 & 3,440 & 3,743 & 4,290 & 5,347 \\
\hline Official current transfers 2/ & 72 & 69 & 82 & 61 & 37 & 34 \\
\hline Private transfers & 2,099 & 2,757 & 3,358 & 3,682 & 4,253 & 5,313 \\
\hline Of which: Workers' remittances & 1,882 & 2,501 & 3,062 & 3,372 & 3,848 & 4,802 \\
\hline Current account balance & $-1,018$ & 157 & 176 & 176 & -557 & 572 \\
\hline Capital and financial account balance & 892 & 808 & 841 & 165 & 947 & 218 \\
\hline Capital account & 432 & 410 & 428 & 196 & 163 & 242 \\
\hline Financial account & 460 & 398 & 413 & -31 & 784 & -24 \\
\hline Foreign direct investment & 174 & 391 & 376 & 276 & 800 & 675 \\
\hline Portfolio investment & 0 & -6 & 2 & 6 & 0 & 32 \\
\hline Net aid flows & 427 & 541 & 466 & 147 & 491 & 432 \\
\hline Aid disbursements $3 /$ & 865 & 963 & 918 & 544 & 940 & 921 \\
\hline Debt amortization & -438 & -422 & -452 & -397 & -449 & -489 \\
\hline Other long-term loans (net) & -13 & -42 & -20 & -41 & -46 & -58 \\
\hline Other short-term loans (net) & 86 & 20 & 142 & 13 & 241 & -256 \\
\hline Other assets (net) & -68 & -52 & -125 & -125 & -182 & -245 \\
\hline Trade credits (net) & -260 & -482 & -499 & -321 & -320 & -805 \\
\hline Commercial banks (net) & 114 & 27 & 71 & 14 & -200 & 201 \\
\hline Errors and omissions & -100 & -557 & -202 & -170 & -323 & -425 \\
\hline Overall balance & -226 & 408 & 815 & 171 & 67 & 365 \\
\hline Financing items & 226 & -408 & -815 & -171 & -67 & -365 \\
\hline Bangladesh Bank 4/ & 226 & -408 & -815 & -171 & -67 & -365 \\
\hline Financing gap & 0 & 0 & 0 & 0 & 0 & 0 \\
\hline \multicolumn{7}{|l|}{ Memorandum items: } \\
\hline Current account balance (percent of GDP) & -2.2 & 0.3 & 0.3 & 0.3 & -0.9 & 0.9 \\
\hline Export growth rate (percent) & 12.6 & -7.6 & 9.5 & 15.9 & 14.0 & 21.6 \\
\hline Import growth rate (percent) & 11.4 & -8.7 & 13.1 & 13.0 & 20.6 & 12.1 \\
\hline Gross official reserves (US\$ million) 5/ & 1,306 & 1,582 & 2,471 & 2,714 & 2,930 & 3,471 \\
\hline (In months of imports of goods and services) & 1.6 & 2.1 & 2.9 & 2.8 & 2.5 & 2.7 \\
\hline Net international reserves (US\$ million) & 447 & 792 & 1,604 & 1,915 & 2,046 & 2,221 \\
\hline Medium and long-term external public debt (US\$ million) & 15,074 & 16,276 & 17,411 & 18,511 & 19,286 & 19,266 \\
\hline (In percent of GDP) & 32.1 & 34.2 & 33.7 & 32.7 & 32.0 & 31.0 \\
\hline Debt-service ratio 6/ & 6.4 & 6.1 & 5.8 & 4.7 & 4.7 & 4.1 \\
\hline Nominal GDP (US\$ million) & 46,998 & 47,565 & 51,719 & 56,554 & 60,299 & 62,016 \\
\hline
\end{tabular}

Sources: Data provided by the Bangladesh authorities; and IMF staff estimates.

1/ Fiscal year begins July 1. Following an STA technical assistance mission in August 2002, presentation of the data has been modified to comply with the fifth edition of the Balance of Payments Manual .

2/ Excludes official capital grants.

3/ Loans only.

4/ Includes Asian Clearing Union balances.

5/ Gross foreign reserves of Bangladesh Bank, including resident foreign currency deposits.

$6 /$ In percent of current earnings defined as the sum of exports of goods, nonfactor services, and private transfers. 
Table 28. Bangladesh: Exports by Main Product Categories, 2000/01-2005/06

2000/01 2001/02 2002/03 2003/04 2004/05 2005/06

\begin{tabular}{lrrrrrr}
\hline Raw jute (millions of U.S. dollars) & 67 & 61 & 82 & 80 & 96 & 148 \\
Volume (millions of bales) & 2 & 1 & 2 & 2 & 2 & 3 \\
Average unit value & 45 & 48 & 41 & 42 & 42 & 43 \\
Jute goods, excl. carpets (millions of U.S. dollars) & 229 & 242 & 257 & 246 & 307 & 361 \\
Volume (thousands of tons) & 372 & 419 & 436 & 472 & 564 & 615 \\
$\quad$ Average unit value & 616 & 577 & 588 & 521 & 545 & 587 \\
Tea (millions of U.S. dollars) & 22 & 17 & 15 & 16 & 16 & 12 \\
$\quad$ Volume (millions of kgs.) & 18 & 14 & 10 & 12 & 11 & 9 \\
Average unit value & 1 & 1 & 1 & 1 & 1 & 1 \\
Leather and leather products (millions of U.S. dollars) & 254 & 207 & 191 & 211 & 221 & 257 \\
$\quad$ Volume (millions of square feet) & 212 & 183 & 165 & 72 & 60 & 77 \\
Average unit value & 1 & 1 & 1 & 3 & 4 & 3 \\
Frozen shrimps and fish (millions of U.S. dollars) & 363 & 276 & 322 & 390 & 421 & 460 \\
$\quad$ Volume (millions of pounds) & 121 & 88 & 74 & 84 & 96 & 108 \\
Average unit value & 3 & 3 & 4 & 5 & 4 & 4 \\
Ready-made garments (millions of U.S. dollars) & 3,364 & 3,125 & 3,258 & 3,539 & 3,598 & 4,084 \\
$\quad$ Volume (millions of dozens) & 71 & 77 & 83 & 90 & 92 & 109 \\
Average unit value index & 47 & 41 & 39 & 39 & 39 & 38 \\
Knitwear and hosiery products (millions of U.S. dollars) & 1,496 & 1,459 & 1,654 & 2,148 & 2,819 & 3,817 \\
$\quad$ Volume (millions of dozens) & 53 & 63 & 69 & 92 & 120 & 165 \\
$\quad$ Average unit value index & 28 & 23 & 24 & 23 & 23 & 23 \\
Naphtha, furnace oil, and bitumen (millions of U.S. dollars) & 10 & 10 & 0 & 0 & 35 & 88 \\
Chemical products (millions of U.S. dollars) & 68 & 48 & 100 & 121 & 197 & 206 \\
Agricultural products (millions of U.S. dollars) & 18 & 23 & 25 & 41 & 82 & 105 \\
Other exports (millions of U.S. dollars) & 528 & 461 & 588 & 730 & 861 & 988 \\
Total exports (millions of U.S. dollars) 1/ & 6,419 & 5,929 & 6,492 & 7,521 & 8,655 & 10,526 \\
\hline
\end{tabular}

Sources: Bangladesh Bank, Statistics Department; Export Promotion Bureau.

1/ Excludes goods procured in ports and repairs on goods. 
Table 29. Bangladesh: Imports by Main Product Categories, 2000/01-2005/06

(In millions of U.S. dollars)

\begin{tabular}{lrrrrrr}
\hline & $2000 / 01$ & $2001 / 02$ & $2002 / 03$ & $2003 / 04$ & $2004 / 05$ & $2005 / 06$ \\
\hline Total & 8,430 & 7,697 & 8,707 & 9,840 & 11,870 & 13,301 \\
Food grains & 380 & 186 & 409 & 431 & 574 & 418 \\
$\quad$ Rice & 180 & 15 & 211 & 144 & 262 & 117 \\
$\quad$ Wheat & 200 & 171 & 198 & 287 & 312 & 301 \\
Edible oil & 230 & 251 & 364 & 471 & 440 & 473 \\
Oilseeds & 70 & 72 & 64 & 73 & 86 & 90 \\
Petroleum products & 575 & 481 & 620 & 770 & 1,252 & 1,400 \\
Crude petroleum & 273 & 242 & 267 & 252 & 350 & 604 \\
Cotton & 375 & 312 & 393 & 583 & 666 & 742 \\
Staple fiber & 42 & 39 & 41 & 57 & 75 & 76 \\
Yarn & 325 & 283 & 270 & 323 & 393 & 501 \\
Fertilizer & 132 & 107 & 109 & 150 & 332 & 342 \\
Textiles & 1,320 & 1,063 & 1,106 & 1,295 & 1,571 & 1,728 \\
Capital goods & 2,400 & 2,617 & 2,735 & 2,875 & 2,814 & 1,539 \\
Other & 2,308 & 2,044 & 2,329 & 2,560 & 3,316 & 5,389 \\
\hline
\end{tabular}

Sources: Bangladesh Bank. 
Table 30. Bangladesh: Geographical Distribution of Foreign Trade, 1999/00-2005/06

(In percent of total)

\begin{tabular}{|c|c|c|c|c|c|c|c|}
\hline & $1999 / 00$ & $2000 / 01$ & $2001 / 02$ & $2002 / 03$ & $2003 / 04$ & $2004 / 05$ & $2005 / 06$ \\
\hline \multicolumn{8}{|l|}{ Exports } \\
\hline United States & 37 & 36 & 37 & 32 & 26 & 26 & 28 \\
\hline Canada & 2 & 2 & 2 & 2 & 3 & 4 & 4 \\
\hline Western Europe & 48 & 49 & 48 & 54 & 60 & 57 & 56 \\
\hline Eastern Europe & 1 & 1 & 1 & 0 & 0 & 0 & 0 \\
\hline India & 1 & 1 & 1 & 1 & 1 & 1 & 2 \\
\hline Pakistan & 1 & 1 & 1 & 1 & 1 & 1 & 1 \\
\hline Japan & 2 & 1 & 2 & 1 & 1 & 1 & 1 \\
\hline Hong Kong, SAR & 2 & 2 & 2 & 2 & 2 & 1 & 2 \\
\hline Singapore & 0 & 1 & 0 & 1 & 1 & 1 & 1 \\
\hline Iran, Islamic Republic of & 1 & 1 & 1 & 1 & 1 & 1 & 1 \\
\hline China & 0 & 0 & 0 & 0 & 0 & 1 & 1 \\
\hline Other & 5 & 6 & 7 & 5 & 4 & 6 & 3 \\
\hline Total & 100 & 100 & 100 & 100 & 100 & 100 & 100 \\
\hline \multicolumn{8}{|l|}{ Imports } \\
\hline United States & 4 & 3 & 3 & 2 & 2 & 3 & 3 \\
\hline Canada & 1 & 1 & 1 & 1 & 1 & 1 & 1 \\
\hline Western Europe & 13 & 13 & 13 & 11 & 11 & 12 & 11 \\
\hline Eastern Europe & 3 & 0 & 1 & 1 & 1 & 2 & 2 \\
\hline India & 10 & 17 & 12 & 15 & 16 & 17 & 14 \\
\hline Pakistan & 1 & 1 & 1 & 1 & 1 & 1 & 1 \\
\hline China & 7 & 10 & 10 & 10 & 12 & 13 & 15 \\
\hline Hong Kong, SAR & 5 & 7 & 5 & 5 & 4 & 5 & 5 \\
\hline Singapore & 8 & 12 & 10 & 11 & 9 & 7 & 6 \\
\hline Korea, Republic of & 4 & 6 & 4 & 4 & 4 & 3 & 4 \\
\hline Malaysia & 1 & 2 & 2 & 2 & 3 & 2 & 2 \\
\hline Indonesia & 10 & 3 & 2 & 2 & 2 & 2 & 2 \\
\hline Thailand & 2 & 3 & 2 & 2 & 2 & 3 & 2 \\
\hline Saudi Arabia & 2 & 2 & 2 & 2 & 2 & 2 & 3 \\
\hline Australia & 2 & 3 & 2 & 2 & 2 & 2 & 2 \\
\hline Japan & 8 & 5 & 8 & 7 & 6 & 5 & 5 \\
\hline Other & 19 & 13 & 23 & 22 & 22 & 20 & 22 \\
\hline Total & 100 & 100 & 100 & 100 & 100 & 100 & 100 \\
\hline
\end{tabular}

Source: Bangladesh Bank, Statistics Department. 
Table 31. Bangladesh: Worker's Remittances by Country of Origin, 2001/02-2006/07 (In millions of U.S. dollars)

\begin{tabular}{|c|c|c|c|c|c|c|}
\hline & $2001 / 02$ & $2002 / 03$ & $2003 / 04$ & $2004 / 05$ & $2005 / 06$ & $2006 / 071 /$ \\
\hline Bahrain & 54 & 64 & 61 & 67 & 67 & 56 \\
\hline Germany & 6 & 10 & 12 & 10 & 12 & 11 \\
\hline Iran, Islamic Republic of & 0 & 0 & 0 & 1 & 2 & 2 \\
\hline Iraq & 0 & 0 & 0 & 0 & 0 & 0 \\
\hline Japan & 14 & 18 & 19 & 16 & 9 & 7 \\
\hline Kuwait & 286 & 339 & 361 & 407 & 494 & 494 \\
\hline Libya & 0 & 0 & 0 & 0 & 0 & 3 \\
\hline Malaysia & 47 & 41 & 37 & 26 & 21 & 9 \\
\hline Oman & 103 & 114 & 119 & 131 & 165 & 146 \\
\hline Qatar & 91 & 114 & 114 & 136 & 176 & 170 \\
\hline Saudi Arabia & 1,148 & 1,254 & 1,386 & 1,510 & 1,697 & 1,312 \\
\hline United Arab Emirates & 233 & 327 & 373 & 442 & 561 & 559 \\
\hline United Kingdom & 103 & 220 & 298 & 376 & 556 & 657 \\
\hline United States & 356 & 458 & 468 & 557 & 761 & 657 \\
\hline Others & 60 & 103 & 124 & 168 & 280 & 279 \\
\hline Total & 2,501 & 3,062 & 3,372 & 3,848 & 4,802 & 4,361 \\
\hline
\end{tabular}

Source: Bangladesh Bank, Statistics Department.

1/ First three quarters. 
Table 32. Bangladesh: Services Account, 2000/01-2005/06 1/

(In millions of U.S. dollars)

\begin{tabular}{lrrrrrr}
\hline & $2000 / 01$ & $2001 / 02$ & $2002 / 03$ & $2003 / 04$ & $2004 / 05$ & $2005 / 06$ \\
& & & & & & \\
& & & & & & \\
& $-1,178$ & -901 & $-1,049$ & $-1,248$ & $-1,549$ & $-1,896$ \\
Services (net) & & & & & & \\
& -914 & -499 & -691 & -874 & -869 & $-1,110$ \\
Nonfactor services (net) & & & & & & \\
& 759 & 865 & 887 & 924 & 1,177 & 1,296 \\
Receipts & 79 & 77 & 87 & 70 & 95 & 109 \\
$\quad$ Transportation & 49 & 54 & 55 & 65 & 68 & 76 \\
$\quad$ Travel & 631 & 734 & 745 & 789 & 1,014 & 1,111 \\
$\quad$ Other & $-1,673$ & $-1,364$ & $-1,578$ & $-1,798$ & $-2,046$ & $-2,406$ \\
& $-1,064$ & -983 & $-1,125$ & $-1,257$ & $-1,504$ & $-1,647$ \\
Payments & -266 & -88 & -134 & -170 & -161 & -138 \\
$\quad$ Transportation & -344 & -293 & -319 & -371 & -381 & -621 \\
$\quad$ Travel & & & & & & \\
$\quad$ Other & -264 & -402 & -358 & -374 & -680 & -786 \\
$\quad$ Investment income (net) & 97 & 50 & 64 & 63 & 116 & 134 \\
$\quad$ Receipts & -361 & -452 & -422 & -438 & -796 & -920 \\
Payments & -159 & -161 & -167 & -175 & -203 & -201 \\
$\quad$ Interest payments & -202 & -291 & -255 & -263 & -593 & -719 \\
$\quad$ Profits and dividends & & & & & & \\
$\quad$ & & & & &
\end{tabular}

Source: Bangladesh Bank.

1/ Reclassified as per recommendations of the IMF Multi-Sector Statistics Mission, August 2002. 
Table 33. Bangladesh: External Aid Commitments and Disbursements, 1999/00-2005/06

(In million U.S. dollars)

\begin{tabular}{|c|c|c|c|c|c|c|c|}
\hline & 1999/00 & $2000 / 01$ & $2001 / 02$ & $2002 / 03$ & $2003 / 04$ & $2004 / 05$ & $2005 / 06$ \\
\hline \multicolumn{8}{|l|}{ Food aid } \\
\hline Opening pipeline & 211 & 55 & 250 & 265 & 225 & 200 & 173 \\
\hline Commitments & 51 & 245 & 51 & 9 & 6 & 6 & 15 \\
\hline Disbursements & 142 & 51 & 36 & 48 & 32 & 33 & 97 \\
\hline Grants 1/ & 142 & 51 & 36 & 48 & 32 & 33 & 97 \\
\hline Loans & 0 & 0 & 0 & 0 & 0 & 0 & 0 \\
\hline Adjustment 2/ & -65 & 0 & 0 & 0 & 0 & 0 & 0 \\
\hline Closing pipeline & 55 & 250 & 265 & 226 & 200 & 173 & 90 \\
\hline \multicolumn{8}{|l|}{ Commodity aid } \\
\hline Opening pipeline & 155 & 40 & 75 & 64 & 12 & 12 & 12 \\
\hline Commitments & 175 & 222 & 144 & 123 & 0 & 22 & 0 \\
\hline Disbursements & 283 & 184 & 155 & 175 & 0 & 22 & 0 \\
\hline Grants 1/ & 280 & 145 & 155 & 175 & 0 & 22 & 0 \\
\hline Loans & 3 & 39 & 0 & 0 & 0 & 0 & 0 \\
\hline Adjustment 2/ & -7 & 0 & -1 & 0 & 0 & 0 & 0 \\
\hline Closing pipeline & 40 & 79 & 64 & 12 & 12 & 12 & 12 \\
\hline \multicolumn{8}{|l|}{ Project aid } \\
\hline Opening pipeline & 5,615 & 5,657 & 5,533 & 5,121 & 5,971 & 6,699 & 6,510 \\
\hline Commitments & 1,254 & 1,585 & 684 & 2,048 & 1,917 & 1,553 & 1,773 \\
\hline Disbursements & 1,150 & 1,134 & 1,251 & 1,362 & 1,002 & 1,434 & 1,470 \\
\hline Grants 3/ & 304 & 309 & 288 & 287 & 307 & 190 & 403 \\
\hline Loans & 846 & 825 & 963 & 1,075 & 695 & 1,244 & 1,067 \\
\hline Adjustment 2/ & -72 & -204 & 155 & 164 & 310 & -309 & -225 \\
\hline Closing pipeline & 5,647 & 5,904 & 5,121 & 5,971 & 7,197 & 6,510 & 6,587 \\
\hline \multicolumn{8}{|l|}{ Total Aid } \\
\hline Opening pipeline & 5,980 & 5,752 & 5,858 & 5,450 & 6,209 & 6,912 & 6,695 \\
\hline Commitments & 1,481 & 2,053 & 879 & 2,179 & 1,923 & 1,581 & 1,787 \\
\hline Disbursements & 1,575 & 1,369 & 1,442 & 1,585 & 1,033 & 1,489 & 1,568 \\
\hline Grants & 726 & 504 & 479 & 510 & 338 & 244 & 501 \\
\hline Loans & 849 & 865 & 963 & 1,075 & 695 & 1,244 & 1,067 \\
\hline Adjustment 2/ & -143 & -204 & 154 & 164 & 310 & -309 & -225 \\
\hline Closing pipeline & 5,742 & 6,233 & 5,449 & 6,209 & 7,408 & 6,695 & 6,689 \\
\hline
\end{tabular}

Source: Economic Relations Division, Ministry of Finance.

1/ Included in "official current transfers" in the current account in Table 29; from 1996/97 onward, data recorded as "official current transfers" include technical assistance-related inflows and, as a result, exceed the sum of food aid and commodity aid in this table.

$2 /$ Includes cancellation and exchange rate adjustments.

$3 /$ Included in "project aid" in the capital account in Table 29. 
Table 34. Bangladesh: External Aid Disbursements by Donors, 1999/00-2005/06

(In millions of U.S. dollars)

\begin{tabular}{|c|c|c|c|c|c|c|c|}
\hline & $1999 / 00$ & $2000 / 01$ & $2001 / 02$ & $2002 / 03$ & $2003 / 04$ & $2004 / 05$ & $2005 / 06$ \\
\hline Bilateral donors & 785 & 706 & 709 & 667 & 495 & 440 & 406 \\
\hline Australia & 8 & 7 & 0 & 9 & 6 & 6 & 11 \\
\hline Belgium & 0 & 0 & 3 & 0 & 0 & 0 & 0 \\
\hline Canada & 28 & 19 & 18 & 23 & 21 & 8 & 62 \\
\hline China & 18 & 26 & 12 & 0 & 0 & 19 & 33 \\
\hline Denmark & 29 & 5 & 29 & 46 & 20 & 5 & 14 \\
\hline France & 1 & 6 & 20 & 1 & 7 & 0 & 0 \\
\hline Finland & 0 & 0 & 0 & 0 & 0 & 0 & 0 \\
\hline Germany & 21 & 43 & 21 & 6 & 26 & 24 & 15 \\
\hline India & 4 & 20 & 7 & 3 & 4 & 26 & 0 \\
\hline Japan & 390 & 316 & 287 & 243 & 79 & 45 & 31 \\
\hline Kuwait & 8 & 36 & 12 & 33 & 7 & 5 & 9 \\
\hline Netherlands & 28 & 19 & 1 & 9 & 41 & 5 & 13 \\
\hline Norway & 19 & 17 & 9 & 50 & 6 & 4 & 11 \\
\hline Saudi Arabia & 4 & 0 & 7 & 0 & 7 & 0 & 0 \\
\hline Sweden & 20 & 16 & 7 & 15 & 11 & 0 & 2 \\
\hline Switzerland & 4 & 7 & 2 & 4 & 5 & 0 & 0 \\
\hline United Kingdom & 61 & 53 & 21 & 40 & 94 & 85 & 157 \\
\hline United States & 92 & 39 & 20 & 35 & 12 & 8 & 4 \\
\hline U.S.S.R. $1 /$ & 0 & 0 & 0 & 0 & 0 & 0 & 0 \\
\hline Suppliers' credits & 43 & 75 & 229 & 157 & 149 & 161 & 44 \\
\hline Other & 7 & 1 & 3 & 3 & $\ldots$ & 39 & $\ldots$ \\
\hline Multilateral donors & 790 & 663 & 733 & 908 & 538 & 1,049 & 1,162 \\
\hline Asian Development Bank & 283 & 236 & 182 & 207 & 172 & 208 & 265 \\
\hline IDA & 354 & 299 & 323 & 561 & 225 & 696 & 635 \\
\hline European Union & 5 & 32 & 80 & 19 & 21 & 8 & 73 \\
\hline IFAD & 15 & 0 & 12 & 21 & 15 & 9 & 14 \\
\hline UNDP & 7 & 17 & 10 & 38 & 36 & ... & 111 \\
\hline World Food Program & 68 & 1 & 30 & 0 & 0 & 0 & \\
\hline UNICEF & 27 & 49 & 46 & 39 & 30 & 26 & 18 \\
\hline \multicolumn{8}{|l|}{ Ford Foundation } \\
\hline Islamic Development Bank & 16 & 16 & 23 & 12 & 17 & 70 & 25 \\
\hline OPEC Fund & 6 & 8 & 14 & 6 & 10 & 3 & 2 \\
\hline Other & 9 & 4 & 12 & 5 & 12 & 28 & 19 \\
\hline Total aid disbursements & 1,575 & 1,369 & 1,442 & 1,575 & 1,033 & 1,488 & 1,568 \\
\hline
\end{tabular}

Source: Economic Relations Division, Ministry of Finance.

1/ The Baltics, Russia, and other former Soviet Union Countries. 
Table 35: Bangladesh: Outstanding External Debt, 2000/01-2005/06 1/

(In millions of U.S. dollars, end of period)

\begin{tabular}{|c|c|c|c|c|c|c|}
\hline & $2000 / 01$ & $2001 / 02$ & $2002 / 03$ & $2003 / 04$ & $2004 / 05$ & $2005 / 06$ \\
\hline Total public sector debt $2 /$ & 15,734 & 16,276 & 16,455 & 16,761 & 19,286 & 19,420 \\
\hline Medium- and long-term loans & 15,607 & 16,067 & 16,234 & 16,404 & 18,644 & 19,015 \\
\hline Central government & 15,336 & 15,885 & 16,126 & 16,203 & 18,416 & 18,602 \\
\hline Multilateral & 11,335 & 11,598 & 11,797 & 11,465 & 13,945 & 14,447 \\
\hline Bilateral & 3,704 & 3,776 & 3,675 & 3,964 & 3,573 & 3,238 \\
\hline Suppliers' credits & 298 & 512 & 653 & 774 & 898 & 917 \\
\hline Other & 99 & 73 & 74 & 56 & 12 & 3 \\
\hline Aircraft loans & 95 & 69 & 71 & 53 & 12 & 3 \\
\hline Shipping loans & 4 & 4 & 4 & 4 & 0 & 0 \\
\hline IMF /3 & 172 & 109 & 34 & 144 & 216 & 410 \\
\hline \multicolumn{7}{|l|}{ Short-term credits } \\
\hline Petroleum loans & 127 & 209 & 221 & 358 & 641 & 405 \\
\hline Debt service payments & -893 & -915 & -926 & $-1,043$ & $-1,139$ & $-1,458$ \\
\hline IMF 3/ & -95 & -73 & -82 & -38 & -3 & -4 \\
\hline Other & -797 & -842 & -844 & $-1,006$ & $-1,137$ & $-1,454$ \\
\hline Principal payments & -710 & -739 & -757 & -858 & -943 & $-1,255$ \\
\hline IMF 3/ & -86 & -69 & -79 & -36 & 0 & 0 \\
\hline Other & -624 & -670 & -678 & -822 & -943 & $-1,255$ \\
\hline Interest payments & -183 & -176 & -169 & -185 & -197 & -203 \\
\hline IMF 3/ & -9 & -4 & -4 & -2 & -3 & -4 \\
\hline Other & -174 & -172 & -165 & -183 & -194 & -200 \\
\hline \multicolumn{7}{|l|}{ Debt indicators (in percent of GDP) } \\
\hline Outstanding debt & 32 & 34 & 34 & 33 & 32 & 31 \\
\hline Outstanding IMF credit $3 /$ & 0 & 0 & 0 & 0 & 0 & 1 \\
\hline Debt service & 2 & 2 & 2 & 2 & 2 & 2 \\
\hline Debt service to the IMF 3/ & 0 & 0 & 0 & 0 & 0 & 0 \\
\hline In percent of GDP & 0.20 & 0.15 & 0.16 & 0.07 & 0.00 & 0.01 \\
\hline
\end{tabular}

Sources: Economic Relations Division, Ministry of Finance; World Bank; and IMF staff estimates.

1/ Fiscal year ending June 30.

2/ Debt recorded by the Economic Relations Division of the Ministry of Finance. On an original maturity basis.

3/ Excludes the IMF Trust Fund. 
Table 36. Bangladesh: Gross Official Reserves, 2002/03-2006/07

(In millions of U.S. dollars; end of period)

\begin{tabular}{|c|c|c|c|c|c|}
\hline & $2002 / 03$ & $2003 / 04$ & $2004 / 05$ & $2005 / 06$ & March 2007 \\
\hline Gold & 40 & 44 & 48 & 67 & 67 \\
\hline SDRs & 4 & 3 & 1 & 1 & 1 \\
\hline Foreign exchange & 2,427 & 2,665 & 2,881 & 3,403 & 4,125 \\
\hline Total $1 /$ & 2,471 & 2,712 & 2,930 & 3,471 & 4,193 \\
\hline
\end{tabular}

Sources: Bangladesh Bank, Statistics Department, and IMF staff estimates.

1/ The figures for total reserves may differ from those presented in the official balance of payments because of valuation differences. 
Table 37. Bangladesh: Summary of the Tax System as of January 2007 (continued)

\begin{tabular}{|c|c|c|c|c|}
\hline \multirow[b]{2}{*}{ Tax } & \multirow[b]{2}{*}{ Nature of Tax } & \multicolumn{3}{|c|}{ Current Tax Status } \\
\hline & & Exemptions and Deduction & & \\
\hline \multirow[t]{23}{*}{ 1.2. Companies } & $\begin{array}{l}\text { Tax is imposed on income of companies } \\
\text { under a classical system of taxation. }\end{array}$ & $\begin{array}{l}\text { Minimum tax ceiling for private limited companies under self- } \\
\text { assessment Tk 50,000. }\end{array}$ & Corporation tax: & $\begin{array}{l}\text { Marginal rate } \\
\text { in percent }\end{array}$ \\
\hline & Resident companies are taxed on worldwide & & Type of enterprise & \\
\hline & income. Nonresident companies are taxed & Exemptions from business income are: & & \\
\hline & on Bangladeshi income. & $\begin{array}{l}\text { * Donation income of religious and charitable institutions under; } \\
\text { certain conditions; }\end{array}$ & $\begin{array}{l}\text { Banks, financial institutions, } \\
\text { and public autonomous }\end{array}$ & 45 percent \\
\hline & $\begin{array}{l}\text { Loss carry-forward is allowed for a maximum } \\
\text { of } 6 \text { successive assessment years. }\end{array}$ & $\begin{array}{l}\text { * Income of cooperative societies engaged in certain } \\
\text { businesses, particularly if derived from agricultural or } \\
\text { rural credit, cottage industries, etc.; }\end{array}$ & $\begin{array}{l}\text { bodies ( } 15 \text { percent "excess } \\
\text { profits tax" imposed on } \\
\text { banks making profit exceeding }\end{array}$ & \\
\hline & $\begin{array}{l}\text { Avoidance of double taxation: } \\
\text { * In the case of residents in Bangladesh, } \\
\text { an ordinary credit (i.e., up to the amount } \\
\text { calculated at the Bangladesh tax rate) is available } \\
\text { with respect to double taxation on income }\end{array}$ & $\begin{array}{l}\text { * Income arising out of poultry, cattle, horticulture, etc., } \\
\text { under certain conditions; } \\
\text { * Agro processing industry up to June 30, } 2008 . \\
\text { * Bonus shares. }\end{array}$ & $\begin{array}{l}50 \text { percent of their capital } \\
\text { and reserve, and additional } \\
5 \text { percent tax on listed } \\
\text { companies declaring inadequate } \\
\text { dividend) }\end{array}$ & \\
\hline & accruing or arising in a foreign country with & Deductions from business income are inter alia allowed for: & & \\
\hline & $\begin{array}{l}\text { which there is no reciprocal arrangement } \\
\text { for the avoidance of double taxation. The } \\
\text { credit is subject to the overall country }\end{array}$ & $\begin{array}{l}\text { * Expenditure incurred wholly and exclusively for the } \\
\text { purposes of the business; } \\
\text { * Bad debts; }\end{array}$ & $\begin{array}{l}\text { Non-publicly traded } \\
\text { companies and local authorities }\end{array}$ & 40 percent \\
\hline & limitation. No relief is available in respect & * Interest payments (no ceilings); & Publicly traded companies & 30 percent \\
\hline & of income which is deemed to accrue or & * Depreciations: There are three categories of depreciation: & & \\
\hline & arise in Bangladesh. & $\begin{array}{l}\text { (i) normal depreciation ( } 10 \text { percent for general buildings, } \\
20 \text { percent for factory buildings, } 20 \text { percent for machinery); }\end{array}$ & $\begin{array}{l}\text { Minimum tax ceiling for } \\
\text { private limited companies }\end{array}$ & $\begin{array}{l}\text { Tax rebate at the } \\
\text { rate of } 10 \text { percent }\end{array}$ \\
\hline & * Double tax treaties apply for several & (ii) accelerated depreciation (which can only be claimed if & under self-assessment is & of tax payable will \\
\hline & $\begin{array}{l}\text { countries (Belgium, Canada, China, } \\
\text { Denmark, France, Germany, India, Italy, }\end{array}$ & $\begin{array}{l}\text { the company is eligible for a tax holiday but chooses to } \\
\text { forego the holiday exemptions): }\end{array}$ & Tk 50,000. & be allowed to the \\
\hline & Japan, Malaysia, Netherlands, Pakistan, & (iii) investment allowance (for certain vessels and for plant and & 100 percent accelerated & declaring dividend \\
\hline & Poland, Republic of Korea, Romania, & machinery eligible for accelerated depreciation). & depreciation is applied for & of 20 percent \\
\hline & Singapore, Sri Lanka, Sweden, Thailand, & * Scientific research expenses. & plants and machineries & or more. \\
\hline & Great Britain \& Northern Ireland, Vietnam, & & with environment protection & \\
\hline & the Philippines, Turkey, and Norway) & $\begin{array}{l}\text { Exporters have a tax rebate of } 50 \text { percent of the income from } \\
\text { exports. Tax rebates of 5-10 percent is allowed for owners of }\end{array}$ & purposes. & \\
\hline & & $\begin{array}{l}\text { small and cottage industries located in less developed and least } \\
\text { developed areas on the basis of production performance. }\end{array}$ & Capital gains tax: & $\begin{array}{l}15 \text { percent, } \\
\text { regardless of the }\end{array}$ \\
\hline & Inter-corporate dividends between & & & retention period of \\
\hline & $\begin{array}{l}\text { Bangladeshi companies are chargeable at a } \\
\text { concessional rate of } 15 \text { percent. }\end{array}$ & $\begin{array}{l}\text { All sources of income of NGOs now subject to taxation } \\
\text { except that arising from micro-credit operations. }\end{array}$ & & assets. \\
\hline & $\begin{array}{l}\text { Tax holiday facilities available for industrial } \\
\text { undertakings, tourist industry, and physical } \\
\text { infrastructure facilities up to June } 30,2008 \text {. }\end{array}$ & $\begin{array}{l}\text { Tax holiday facilities for expansion units have been } \\
\text { withdrawn, and only separately incorporated projects will be } \\
\text { eligible for such facility. }\end{array}$ & & \\
\hline & & $\begin{array}{l}\text { Reinvestment limit for companies to continue enjoying } \\
\text { tax holiday facilities has been increased from } 30 \text { percent of } \\
\text { profits to } 40 \text { percent. }\end{array}$ & & \\
\hline
\end{tabular}

CInternational Monetary Fund. Not for Redistribution 
Table 37. Bangladesh: Summary of the Tax System as of January 2007 (continued)

Tax

Nature of Tax
Current Tax Status

Exemptions and Deduction
1.2. Companies
As an alternative to tax holiday 20 percent concessional

corporate tax rate for newly set up industries has been

introduced.

Newly set up industries, depending on situation, have been

given 100 percent accelerated depreciation allowance in the

very first year instead of the first two years. Newly set up

companies (between July 1, 2002 to June 30, 2005)

that would not get tax holiday facilities will pay tax at

reduced rate of 20 percent for five years.

English medium schools, colleges, and universities in

the private sector now subject to tax. However,

medical, dental, and engineering colleges, and

institutions providing education on information technology,

will not be taxed. 
Table 37. Bangladesh: Summary of the Tax System as of January 2007 (continued)

\begin{tabular}{|c|c|c|c|c|}
\hline \multirow[b]{2}{*}{ Tax } & \multirow[b]{2}{*}{ Nature of Tax } & \multicolumn{3}{|c|}{ Current Tax Status } \\
\hline & & Exemptions and Deduction & & \\
\hline 1.5 Gift tax & $\begin{array}{l}\text { Tax imposed on the value of } \\
\text { gifts made by donor. }\end{array}$ & $\begin{array}{l}\text { Exemptions are: } \\
\text { * Gifts under Tk 20,000; } \\
\text { * Assets situated outside of Bangladesh; } \\
\text { * Gifts to the government or local authority; } \\
\text { * Gifts made by will; } \\
\text { * Gifts to institution established for charitable purposes and } \\
\text { recognized by the Government (unto Tk } 100,000 \text { or total income } \\
\text { of donor, whichever is less); } \\
\text { * Insurance policies or annuities to any relative (other than a } \\
\text { wife) supported by the taxpayer with a value not exceeding } \\
\text { Taka } 20,000 \text { per year; } \\
\text { * Gifts made to son, daughter, father, mother, spouse, own } \\
\text { brother, own sister; } \\
\text { * Gifts made to any dependent relative on the occasion of marriage; } \\
\text { * Gifts made in contemplation of death; } \\
\text { * Gifts made to any educational institution recognized by } \\
\text { the Government, universities, or Educational Board; } \\
\text { * Gifts made by Government or local authority to any hospital; } \\
\text { * Flood or disaster-related fund recognized by the Government; } \\
\text { * Gifts made by any statutory institution established under any } \\
\text { law of the country; } \\
\text { * Gifts made by any religious or charitable institution established } \\
\text { under law; } \\
\text { * Gifts made by any charitable institution or made out to any } \\
\text { tax-exempt fund. }\end{array}$ & $\begin{array}{l}\text { Value of gift } \\
\text { Tk 20,001-500,000 } \\
\text { Tk } 500,001-1,500,000 \\
\text { Tk 1,500,001-3,500,001 } \\
\text { Tk } 3,500,001 \text { and above }\end{array}$ & $\begin{array}{l}\text { Marginal rate } \\
\\
5 \text { percent } \\
10 \text { percent } \\
15 \text { percent } \\
20 \text { percent }\end{array}$ \\
\hline
\end{tabular}


Table 37. Bangladesh: Summary of the Tax System as of January 2007 (continued)

\begin{tabular}{|c|c|c|c|}
\hline \multirow[b]{2}{*}{$\operatorname{Tax}$} & \multirow[b]{2}{*}{ Nature of Tax } & \multicolumn{2}{|c|}{ Current Tax Status } \\
\hline & & Exemptions and Deduction & Rates \\
\hline
\end{tabular}

2. Taxes on goods

and services

2.1 Import duties

Ad valorem tax on imported goods.

Special concessional rates apply to spare parts, electrical textiles, pharmaceuticals raw materials, poultry, dairy, solar panel, capital machinery

equipment, agricultural inputs, and other products under agreement.

tant bands with a maxinum rate of 25 percent and a

Export-oriented industry may import machinery duty free.

Non export-oriented industry pay 5 percent custom

duty on machinery import.

Special lower rate for cars imported under the taxicab facility.

Customs duties on imports used to produce exports can be

refunded through the Duty Exemption and Drawback Office (DEDO).

2.2 Export duties

Effective rates of export duty on all commodities are zero.

Ad valorem tax on all imports. There are plans to extend this tax to selected domestic goods.

2.3 Infrastry

surcharge

2.4 VAT

2.4.1 Value-added tax Invoice method VAT applied to manufactures,
imports, and selected services and goods at

\section{Exports are zero-rated.}

VAT is levied on the base inclusive of customs duties and supplementary duties.

Wholesalers and retailers may register for VAT

\section{Exempt are:}

imports of defense stores;

"imports of diplomatic and privileged persons;

"imports for blind-deaf and dumb and other handicapped person

relief goods imported for disaster management;

imports for export purposes.

\section{Exempt are:}

* firms with turnover less than Tk 2 million per annum

4 percent turnover tax is applicable to them.

* education, public administration, housing, and charitable health services,

cold storage travel agency, indenting firm.

Construction faces a reduced tax of 4.5 percent without

credit for invoiced tax

Exempt are the following goods: animals, meat, eggs, hides,

fish, vegetables, fruit, grain, flour, cattle and poultry feed, primary

milk products, insecticides, jute cuttings, oilseeds, a few

chemicals and drugs, fertizers, domestic textles, some

plastics, metal products, electricity used in the agricultural sector,

a wide range of machinery and scientific apparatus.
4 percent

15 percent

Fixed VAT amounted to Tk 4,200 for small retailers of Dhaka and Chittagong

City Corporation areas, TK 3,600 for other city Corporation areas,

areas of the country.

Truncated rates of 2.25 percent, 4.5 percent, 5 percent, and 9 percent in cases where invoice method is difficult to apply.

5.0025 percent for electricity. 
Table 37. Bangladesh: Summary of the Tax System as of January 2007 (continued)




Table 37. Bangladesh: Summary of the Tax System as of January 2007 (concluded)

\begin{tabular}{|c|c|c|c|}
\hline \multirow[b]{2}{*}{ Tax } & \multirow[b]{2}{*}{ Nature of Tax } & \\
\hline & & Exemptions and Deduction & 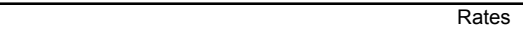 \\
\hline 2.11 Advertisement tax & $\begin{array}{l}\text { Tax imposed on expenses on advertisements made through } \\
\text { cinema slides and films, radio, and television (except for } \\
\text { tenders and employment notices). }\end{array}$ & & 15 percent \\
\hline 2.12 Motor vehicles & $\begin{array}{l}\text { Specific tax on taxicabs and buses based on } \\
\text { seating capacity. }\end{array}$ & & $\begin{array}{l}\text { Type of motor vehicle } \\
\text { Taxicabs and buses carrying } \\
\text { Up to } 4 \text { passengers } \\
4-6 \text { passengers } \\
6-15 \text { passengers } \\
15-30 \text { passengers } \\
\text { Above } 30 \text { passengers } \\
\text { Double decker buses }\end{array}$ \\
\hline 2.13 Irrigation tax & $\begin{array}{l}\text { Water rates are levied in respect of projects completed } \\
\text { by the Water Development Board. }\end{array}$ & & \\
\hline 2.14 Stamp duty & $\begin{array}{l}\text { Levied on the registration value of land, flat etc. at time of } \\
\text { sale or transfer of property }\end{array}$ & & 5 percent \\
\hline \multicolumn{4}{|l|}{ B. Local taxes } \\
\hline 1 Thana vehicles tax & Specific tax imposed on vehicles. & & Rates may vary by thana. \\
\hline 2 Local property taxes & $\begin{array}{l}\text { Property taxes imposed by union parishads on structur Exempt is agricultural land. } \\
\text { and nonagricultural land. Two separate taxes are i imposed, } \\
\text { the union rate and the chowkidari rate (for funding } \\
\text { local police). }\end{array}$ & & $\begin{array}{l}\text { For the union rate, a maximum of } 7 \text { percent of annual } \\
\text { rental value; for the chowkidari rate, a rate sufficient } \\
\text { to cover costs for local law enforcement and the cost of } \\
\text { tax collection. }\end{array}$ \\
\hline
\end{tabular}

